TRANSACTIONS OF THE

AMERICAN MATHEMATICAL SOCIETY

Volume 364, Number 7, July 2012, Pages 3479-3511

S 0002-9947(2012)05428-6

Article electronically published on February 29, 2012

\title{
ON THE SEMIALGEBRAIC STONE-ČECH COMPACTIFICATION OF A SEMIALGEBRAIC SET
}

\author{
JOSÉ F. FERNANDO AND J. M. GAMBOA
}

Dedicated to José María Montesinos on the occasion of his $65^{\text {th }}$ birthday

\begin{abstract}
In the same vein as the classical Stone-Cech compactification, we prove in this work that the maximal spectra of the rings of semialgebraic and bounded semialgebraic functions on a semialgebraic set $M \subset \mathbb{R}^{n}$, which are homeomorphic topological spaces, provide the smallest Hausdorff compactification of $M$ such that each bounded $\mathbb{R}$-valued semialgebraic function on $M$ extends continuously to it. Such compactification $\beta_{s}^{*} M$, which can be characterized as the smallest compactification that dominates all semialgebraic compactifications of $M$, is called the semialgebraic Stone-Čech compactification of $M$, although it is very rarely a semialgebraic set. We are also interested in determining the main topological properties of the remainder $\partial M=\beta_{s}^{*} M \backslash M$ and we prove that it has finitely many connected components and that this number equals the number of connected components of the remainder of a suitable semialgebraic compactification of $M$. Moreover, $\partial M$ is locally connected and its local compactness can be characterized just in terms of the topology of $M$.
\end{abstract}

\section{INTRODUCTION}

A subset $M \subset \mathbb{R}^{n}$ is said to be basic semialgebraic if it can be written as

$$
M=\left\{x \in \mathbb{R}^{n}: f(x)=0, g_{1}(x)>0, \ldots, g_{m}(x)>0\right\}
$$

for some polynomials $f, g_{1}, \ldots, g_{m} \in \mathbb{R}\left[\mathrm{x}_{1}, \ldots, \mathrm{x}_{n}\right]$. The finite unions of basic semialgebraic sets are called semialgebraic sets. A continuous function $f: M \rightarrow \mathbb{R}$ is said to be semialgebraic if its graph is a semialgebraic subset of $\mathbb{R}^{n+1}$. Usually, semialgebraic function just means a function, not necessarily continuous, whose graph is semialgebraic. However, since all semialgebraic functions occurring in this article are continuous we will omit for simplicity the continuity condition when we refer to them.

The sum and product of functions, defined pointwise, endow the set $\mathcal{S}(M)$ of semialgebraic functions on $M$ with a natural structure of commutative ring whose unity is the function with constant value 1 . In fact, $\mathcal{S}(M)$ is an $\mathbb{R}$-algebra if we identify each real number $r$ with the constant function which just attains this value. The most simple examples of semialgebraic functions on $M$ are the restrictions to

Received by the editors June 4, 2010.

2010 Mathematics Subject Classification. Primary 14P10, 54C30; Secondary 12D15, 13E99.

Key words and phrases. Semialgebraic function, maximal spectrum, semialgebraic compactification, semialgebraic Stone-Čech compactification, remainder.

Both authors were supported by Spanish GAAR MTM2011-22435, Proyecto Santander Complutense PR34/07-15813 and GAAR Grupos UCM 910444. 
$M$ of polynomials in $n$ variables. Other relevant ones are the absolute value of a semialgebraic function, the maximum and the minimum of a finite family of semialgebraic functions, and the inverse and the $k$-root of a semialgebraic function whenever these operations are well defined.

It is obvious that the subset $\mathcal{S}^{*}(M)$ of bounded semialgebraic functions on $M$ is a real subalgebra of $\mathcal{S}(M)$. For the time being we denote by $\mathcal{S}^{\diamond}(M)$, indistinctly, either $\mathcal{S}(M)$ or $\mathcal{S}^{*}(M)$ in case the involved statements or arguments are valid for both rings.

The first remarkable fact concerning the maximal spectra of these rings is that the respective maximal spectra $\beta_{\mathrm{s}} M$ and $\beta_{\mathrm{s}}^{*} M$ of $\mathcal{S}(M)$ and $\mathcal{S}^{*}(M)$ are homeomorphic (see 3.5). This phenomenon occurs identically for rings of continuous functions on a completely regular topological space, although the techniques involved to prove such facts are substantially different (see for instance [GJ] §6- $\$ 7]$ ). The homeomorphism between $\beta_{\mathrm{s}} M$ and $\beta_{\mathrm{s}}^{*} M$ constructed in 3.5 allows us to characterize algebraically those maximal ideals of the rings $\mathcal{S}^{\diamond}(M)$ corresponding to a point in $M$ (see 3.7 and 3.11) and the compactness of $M$ in terms of the equality of the rings $\mathcal{S}(M)$ and $\mathcal{S}^{*}(M)$.

In the same vein as the classical Stone-Čech compactification, we prove (see 4.4 and 4.77) that $\beta_{\mathrm{s}}^{*} M$ is the smallest compactification of $M$ such that each bounded $\mathbb{R}$ valued semialgebraic function on $M$ extends continuously to $\beta_{\mathrm{s}}^{*} M$. This is why we will call $\beta_{\mathrm{s}}^{*} M$ the semialgebraic Stone-Čech compactification of $M$, although $\beta_{\mathrm{s}}^{*} M$ is very rarely (homeomorphic to) a semialgebraic set. In fact, we characterize in 5.17 the semialgebraic sets $M$ such that $\beta_{\mathrm{s}}^{*} M$ is a semialgebraic set, as those whose subset of points of local dimension $\geq 2$ is compact. Note that for a 1-dimensional $M$ the compactification $\beta_{\mathrm{s}}^{*} M$ is obtained by adding to $M$ an ending point at each open half-branch (see 4.20). For instance, if $M=[0,1)$ or $(0,1)$, then $\beta_{\mathrm{s}}^{*} M=[0,1]$ (see 4.9).

Furthermore, the semialgebraic Stone-Čech compactification of $M$ is the smallest compactification that dominates each semialgebraic compactification of $M$ and, in fact, it is decisive to realize that $\beta_{\mathrm{s}}^{*} M$ is the "inverse limit" of the collection of such semialgebraic compactifications of $M$ (see 4.6). These results suggest that the topology of $\beta_{\mathrm{s}}^{*} M$ can be recovered from the semialgebraic compactifications of $M$. We are interested in determining the main topological properties of the remainder $\partial M=\beta_{\mathrm{s}}^{*} M \backslash M$, and we prove (see 5.8 ) that it has finitely many connected components and that in fact this number equals the number of connected components of the remainder of a suitable semialgebraic compactification of $M$. In any case, the number of connected components of $\partial M$ upperly bounds the number of connected components of the remainder $X \backslash M$ of any semialgebraic compactification $X$ of $M$ (see 5.2). Other remarkable properties of $\partial M$ are its local connectedness (see 5.13) and that the topology of $M$ determines its local compactness (see 5.14).

This article is organized as follows. In Section 2 we collect most of the preliminary definitions, notation and results that will be used freely in the sequel. It is worthwhile mentioning 2.9 which is a technical refinement of the classical theorem BCR, 9.2.1] concerning triangulations of compact semialgebraic subsets of $\mathbb{R}^{n}$, and this will be crucial for the proof of 5.8. Next, in Section 3 we develop the algebraic approach to the homeomorphism between $\beta_{\mathrm{s}} M$ and $\beta_{\mathrm{s}}^{*} M$ mentioned above, while in Section 4 we analyze this space $\beta_{\mathrm{s}}^{*} M$ from the topological point of view. Finally, 
Section 5 is devoted to the study of some of the most remarkable topological properties of the remainder $\partial M$. Also in this section, we prove that the space $\beta_{\mathrm{s}}^{*} M$ is rarely (homeomorphic to) a semialgebraic set and we study under what conditions the operator $\beta_{\mathrm{s}}^{*}$ commutes with finite products.

To finish this Introduction, we point out that our guideline to initially approach this work has been GJ], and many statements have been proposed after properly adapting the corresponding ones for rings of continuous functions on a completely regular topological space. However, the advantageous conditions of rings of semialgebraic functions on a semialgebraic set allow us to achieve sharper results by using specific techniques of semialgebraic geometry.

\section{Preliminaries on Semialgebraic Sets AND Semialgebraic FunCtions}

To begin this section we introduce some terminology, notation and preliminary results that will be used systematically in this work. For each $f \in \mathcal{S}^{\diamond}(M)$ and each semialgebraic subset $N \subset M$, we denote $Z_{N}(f)=\{x \in N: f(x)=0\}$ and $D_{N}(f)=N \backslash Z_{N}(f)$. In case $N=M$, we say that $Z_{M}(f)$ is the zeroset of $f$. We respectively denote by $\mathbb{B}_{n}(x, \varepsilon)$ and $\overline{\mathbb{B}}_{n}(x, \varepsilon)$ the open and closed balls of $\mathbb{R}^{n}$ of center $x$ and radius $\varepsilon$, while their common boundary is denoted by $\mathbb{S}^{n-1}(x, \varepsilon)$. Sometimes it will be useful to assume that the semialgebraic set $M$ we are working with is bounded. Such an assumption can be done without loss of generality. Namely,

Remark 2.1. Let $M \subset \mathbb{R}^{n}$ be a semialgebraic set. The semialgebraic homeomorphism

$$
\varphi: \mathbb{B}_{n}(0,1) \rightarrow \mathbb{R}^{n}, x \mapsto \frac{x}{\sqrt{1-\|x\|^{2}}}
$$

induces a ring isomorphism $\mathcal{S}(M) \rightarrow \mathcal{S}(N), f \mapsto f \circ \varphi$, where $N=\varphi^{-1}(M)$, that maps $\mathcal{S}^{*}(M)$ onto $\mathcal{S}^{*}(N)$. Hence, if necessary, we may always assume that $M$ is bounded.

The next result, which concerns the representation of closed semialgebraic subsets of a semialgebraic set as zerosets of semialgebraic functions, is well known and will be used freely in this work (see for instance [FG1, 2.2]).

Lemma 2.2. Let $Z$ be a closed semialgebraic subset of a semialgebraic set $M \subset \mathbb{R}^{n}$. Then, there exists $h \in \mathcal{S}^{*}(M)$ such that $Z=Z_{M}(h)$.

As a crucial ingredient in many proofs of this work, here we recall the following semialgebraic version of the Tietze-Urysohn Lemma due to Delfs and Knebusch (see $[\overline{D K}]$ ) that will be used freely in what follows.

Theorem 2.3. Let $N \subset M \subset \mathbb{R}^{n}$ be semialgebraic sets such that $N$ is closed in $M$. Then, the homomorphism $\mathcal{S}^{\diamond}(M) \rightarrow \mathcal{S}^{\diamond}(N),\left.F \mapsto F\right|_{N}$ is surjective.

As a straightforward consequence, we have the following:

Corollary 2.4. Let $C_{1}, C_{2} \subset M \subset \mathbb{R}^{n}$ be semialgebraic sets such that $C_{1}$ and $C_{2}$ are closed disjoint subsets of $M$. Then, there exists $f \in \mathcal{S}^{*}(M)$ such that $\left.f\right|_{C_{1}} \equiv 0$ and $\left.f\right|_{C_{2}} \equiv 1$.

Next, we present the triangulability of semialgebraic sets, which is one of the most powerful tools to approach topological properties of this kind of sets. Moreover, we include some results about triangulations that will be needed in forthcoming sections. 
(2.5) Triangulations of semialgebraic sets. Let $P_{0}, \ldots, P_{k}$ be $k+1$ affinely independent points in $\mathbb{R}^{n}$. The $k$-simplex $\left[P_{0}, \ldots, P_{k}\right]$ is the set of those $x \in \mathbb{R}^{n}$ such that there exist nonnegative real numbers $\lambda_{0}, \ldots, \lambda_{k}$ with $\sum_{i=0}^{k} \lambda_{i}=1$ and $x=\sum_{i=0}^{k} \lambda_{i} P_{i}$. Of course, the $k$-simplex $\left[P_{0}, \ldots, P_{k}\right]$ is a compact semialgebraic manifold with boundary, semialgebraically homeomorphic to the closed ball $\overline{\mathbb{B}}_{k}(0,1)$ of $\mathbb{R}^{k}$. Next, if $\left\{P_{i_{0}}, \ldots, P_{i_{\ell}}\right\}$ is a nonempty subset of $\left\{P_{0}, \ldots, P_{k}\right\}$, then the $\ell$ simplex $\left[P_{i_{0}}, \ldots, P_{i_{\ell}}\right]$ is called an $\ell$-face of $\left[P_{0}, \ldots, P_{k}\right]$. If $\sigma$ is a simplex, then the open simplex $\sigma^{0}$ is the subset of points of $\sigma$ not belonging to any proper face of $\sigma$. Note that $\mathrm{Cl}_{\mathbb{R}^{n}}\left(\sigma^{0}\right)=\sigma$ and that $\sigma=\sigma^{0}$ just in case $\sigma$ is a point.

A finite simplicial complex of $\mathbb{R}^{n}$ is a finite collection of simplices $K=\left(\sigma_{i}\right)_{i=1}^{p}$ such that the faces of every $\sigma_{i}$ belong to $K$ and such that, for every $1 \leq i, j \leq p$, the intersection $\sigma_{i} \cap \sigma_{j}$ is either empty or a common face of $\sigma_{i}$ and $\sigma_{j}$. A straightforward computation shows that $\sigma_{i}^{0} \cap \sigma_{j}$ is either empty or $\sigma_{i}^{0}$. The realization of the complex $K$ is $|K|=\bigcup_{i=1}^{p} \sigma_{i}=\bigcup_{i=1}^{p} \sigma_{i}^{0}$; in fact, the open simplices $\sigma_{i}^{0}$ constitute a partition of $|K|$. A semialgebraic triangulation of a compact semialgebraic set $X \subset \mathbb{R}^{n}$ is a pair $(K, \Phi)$, where $K$ is a finite simplicial complex and $\Phi:|K| \rightarrow X$ is a semialgebraic homeomorphism. The triangulation $(K, \Phi)$ of $X$ is said to be compatible with a finite family $\mathcal{F}$ of semialgebraic subsets of $X$ if each set $S \in \mathcal{F}$ is the union of some $\Phi\left(\sigma^{0}\right)$ with $\sigma \in K$.

2.51) We recall a well-known procedure to triangulate a $d$-dimensional simplex $\sigma$ from a triangulation of one of its $(d-1)$-dimensional faces $\tau$. Observe that there exists a unique vertex $P$ of $\sigma$ which is not contained in $\tau$. This way, each triangulation $K$ of $\tau$ induces a triangulation $\widehat{K}$ of $\sigma$ defined as follows. For each simplex $\eta \in K$ denote by $\widehat{\eta}$ the simplex generated by $\eta$ and $P$. Then, $\widehat{K}$ is the triangulation of $\sigma$ whose simplices are the simplices $\widehat{\eta}$ generated by $\eta$ and $P$, the simplices of $K$ and $\{P\}$.

The fundamental result about semialgebraic triangulations of compact semialgebraic sets is the following theorem (see [BCR, 9.2.1]).

Theorem 2.6 (Semialgebraic triangulation). Let $X \subset \mathbb{R}^{n}$ be a compact semialgebraic set and let $\mathcal{F}$ be a finite family of semialgebraic subsets of $X$. Then, there exists a semialgebraic triangulation of $X$ compatible with $\mathcal{F}$.

A particular case to be considered consists of a semialgebraic set $X \subset \mathbb{R}^{n}$ which is itself a simplex, and whose trivial triangulation is the one whose simplices are $X$ and all its faces. For later purposes (see [5.8), we need more sophisticated triangulations of a compact semialgebraic set than the one provided in 2.6. More precisely,

Lemma 2.7. Let $M \subset X \subset \mathbb{R}^{n}$ be semialgebraic sets such that $X$ is compact. Let $\mathcal{F}$ be a finite family of semialgebraic subsets of $X$ containing $M$. Then, there exists a semialgebraic triangulation $(K, \Phi)$ of $X$ compatible with $\mathcal{F}$ such that for each simplex $\sigma \in K$ either $\sigma \subset \Phi^{-1}(M)$ or there exists a face $\tau$ of $\sigma$ satisfying $\tau^{0} \subset \sigma \backslash \Phi^{-1}(M) \subset \tau$.

Before proving 2.7, we need a preliminary technical result. Namely,

Lemma 2.8. Let $X \subset \mathbb{R}^{n}$ be an n-dimensional simplex and let $M$ be a union of some open faces of $X$ such that for each face $\sigma$ of $X$ either $\sigma \subset M$ or there exists a face $\tau$ of $\sigma$ such that $\tau^{0} \subset \sigma \backslash M \subset \tau$. Let $K$ be a triangulation of $X$ compatible with the open faces of $X$. Then, for all $\theta \in K$, either $\theta \subset M$ or there exists a face $\zeta$ of $\theta$ such that $\zeta^{0} \subset \theta \backslash M \subset \zeta$. 
Proof. We proceed by induction on the dimension $d$ of $\theta$. If $\operatorname{dim} \theta=0$, then $\theta$ is a singleton, and so, either $\theta \subset M$ or $\theta^{0}=\theta \backslash M=\theta$ if $\theta \not \subset M$. Assume that for each $k$-dimensional simplex $\theta \in K$ with $k \leq d-1$, either $\theta \subset M$ or there exists a face $\zeta$ of $\theta$ such that $\zeta^{0} \subset \theta \backslash M \subset \zeta$, and let us check that the same holds for the $d$-dimensional simplices of the complex $K$.

Indeed, let $\theta \in K$ be a $d$-dimensional simplex such that $\theta \not \subset M$. Let $\sigma$ be the unique face of $X$ such that $\theta^{0} \subset \sigma^{0}$. Since $\theta \not \subset M$, also $\sigma \not \subset M$, and so there exists a face $\tau$ of $\sigma$ such that $\tau^{0} \subset \sigma \backslash M \subset \tau$. Observe that $\theta \backslash M=\theta \cap(\sigma \backslash M) \subset(\theta \cap \tau) \backslash M$. Since $K$ is compatible with $\tau$ and $\theta \backslash M \neq \varnothing$, the intersection $\theta \cap \tau$ is the union of the relative interiors of some faces of $\theta$. Since $\theta$ and $\tau$ are compact and convex, so is its intersection $\theta \cap \tau$. Thus, $\theta \cap \tau$ is the union of some faces of $\theta$; let $P_{1}, \ldots, P_{s}$ be all the vertices of $\theta$ which belong to $\tau$. Since $\theta \cap \tau$ is the union of some faces of $\theta$ and it is convex, we deduce that $\varrho=\theta \cap \tau$ is the simplex $\left[P_{1}, \ldots, P_{s}\right] \in K$. Now, we distinguish two cases:

Case 1. If $\operatorname{dim} \varrho=d$, then $\varrho=\theta$ and so $\theta \subset \tau$. Hence, $\varnothing \neq \theta^{0} \subset \tau \cap \sigma^{0}$ and therefore $\tau=\sigma$. Thus, $\sigma^{0} \subset \sigma \backslash M$ and we deduce

$$
\theta^{0}=\theta^{0} \cap \sigma^{0} \subset \theta \cap(\sigma \backslash M)=\theta \backslash M \subset \theta,
$$

which implies $\theta^{0} \subset \theta \backslash M \subset \theta$.

Case 2. If $\operatorname{dim} \varrho<d$, then, by induction hypothesis, there exists a face $\zeta$ of $\varrho$, which is also a face of $\theta$, such that $\zeta^{0} \subset \varrho \backslash M \subset \zeta$. Hence,

$$
\zeta^{0} \subset \varrho \backslash M=(\theta \cap \tau) \backslash M=\theta \cap(\tau \backslash M) \subset \theta \backslash M \subset(\theta \cap \tau) \backslash M=\varrho \backslash M \subset \zeta,
$$

that is, $\zeta^{0} \subset \theta \backslash M \subset \zeta$, as wanted.

Proof of Lemma 2.7. We first study the fundamental case in which $M$ is a finite union of open faces of a simplex $X$ of dimension $d$ and $\mathcal{F}=\{M\}$. We proceed by induction on the dimension of $X$, the case $\operatorname{dim} X=0$ being trivial. If $\operatorname{dim} X=1$, it is enough to choose as $K$ a barycentric subdivision of the trivial triangulation of $X$. Suppose the result is true for $\operatorname{dim} X=d-1$ and let us see that it is also true for $\operatorname{dim} X=d$.

(2.71) First, we choose an initial triangulation $K_{0}$ of $X$ as follows. If $X^{0} \not \subset M$ we consider in $X$ its trivial triangulation $K_{0}$. On the other hand, if $X^{0} \subset M$, we choose a barycentric subdivision of $X$ which provides a triangulation $K_{0}$ of $X$ whose simplices are:

(a) all the proper faces of $X$,

(b) the barycenter $G$ of $X$, and

(c) those simplices $\widehat{\delta}$ generated by $G$ and a proper face $\delta$ of $X$.

Observe that in this second case in which $X^{0} \subset M$, each $d$-dimensional simplex $\sigma$ of $K_{0}$ satisfies one of the following conditions:

(1) $\sigma \subset M$.

(2) There exists a $(d-1)$-dimensional (proper) face $\epsilon$ of $\sigma$, which is also a proper face of $X$, such that $\sigma \backslash M \subset \epsilon$ and $\sigma=\widehat{\epsilon}$ is the simplex generated by $G$ and $\epsilon$.

Of course, $\sigma^{0} \subset X^{0} \subset M$ and the barycenter of $X$ is a point of $X^{0}$. Moreover, each proper face of the simplex $X$ is a face of some of the $(d-1)$-dimensional faces of the simplex $X$. 
(2.72) For each simplex $\vartheta \in K_{0}$, we denote $M_{\vartheta}=M \cap \vartheta$. For each $(d-1)$ dimensional simplex $\nu \in K_{0}$ there exists, by induction hypothesis, a triangulation $K_{\nu}$ of $\nu$ such that for each simplex $\varsigma \in K_{\nu}$ either $\varsigma \subset M_{\nu}$ or there exists a face $\iota$ of $\varsigma$ satisfying $\iota^{0} \subset \varsigma \backslash M_{\nu} \subset \iota$. Next, we distinguish two cases:

Case 1. $X^{0} \not \subset M$. Since $M$ is a finite union of open faces of the simplex $X$, we have $X^{0} \cap M=\varnothing$. By 2.6, there exists a triangulation $K_{1}$ of $X$ compatible with $X^{0}$ and all the open faces of all the simplices in the triangulations $K_{\nu}$ of all the $(d-1)$-dimensional simplices $\nu$ of $K_{0}$. Fix a simplex $\theta \in K_{1}$ such that $\theta \not \subset M$. If $\theta \cap X^{0}=\varnothing$, then $\theta$ is contained in a $(d-1)$-dimensional face $\nu$ of $X$ and, by 2.8. there exists a face $\zeta$ of $\theta$ such that $\zeta^{0} \subset \theta \backslash M \subset \zeta$. On the other hand, if $\theta \cap X^{0} \neq \varnothing$, then also $\theta^{0} \cap X^{0} \neq \varnothing$ and, since $K_{1}$ is compatible with $X^{0}$, we deduce $\theta^{0} \subset X^{0}$. Hence, $\theta^{0} \backslash M=\theta^{0} \cap\left(X^{0} \backslash M\right)=\theta^{0}$ and so $\theta^{0} \subset \theta \backslash M \subset \theta$. Thus, $K_{1}$ has the desired property that for each simplex $\theta \in K_{1}$ either $\theta \subset M$ or there exists a face $\zeta$ of $\theta$ satisfying $\zeta^{0} \subset \theta \backslash M \subset \zeta$.

Case 2. $X^{0} \subset M$. If $\sigma \in K_{0}$ is a $d$-dimensional simplex satisfying condition (1) in 2.711, that is, $\sigma \subset M$, we choose the trivial triangulation $K_{\sigma}$ of $\sigma$. On the other hand, if $\sigma$ satisfies condition (2) in 2.711 we proceed as follows. Recall that $\sigma=\widehat{\epsilon}$ is the simplex generated by the barycenter $G$ and a $(d-1)$-dimensional face $\epsilon$ of $X$ such that $\sigma \backslash M \subset \epsilon$. Let $K_{\epsilon}$ be a triangulation of $\epsilon$ given by the inductive hypothesis. Now, we construct the triangulation $K_{\sigma}=\widehat{K}_{\epsilon}$ of $\sigma=\widehat{\epsilon}$ induced by $K_{\epsilon}$; see 2.511. Let us check that for each simplex $\theta$ of $K_{\sigma}$ :

(2.73) Either $\theta \subset M_{\sigma}$ or there exists a proper face $\zeta$ of $\theta$ such that $\zeta^{0} \subset \theta \backslash M_{\sigma} \subset \zeta$.

Indeed, by the properties of $K_{\epsilon}$, the claim of 2.713 is true if $\theta \subset \epsilon$ because $M_{\epsilon}=M_{\sigma} \cap \epsilon$. Moreover, since $G \in X^{0} \cap \sigma \subset M \cap \sigma=M_{\sigma}$, then $\{G\} \subset M_{\sigma}$. Thus, we may assume that $\theta \not \subset M_{\sigma}$ and $\theta=\widehat{\varrho}$ for some $\varrho \in K_{\epsilon}$. In this case, since $G \in X^{0}$, we have $\theta \backslash \varrho \subset X^{0} \cap \sigma \subset M \cap \sigma=M_{\sigma}$. Thus, $\theta \backslash M_{\sigma} \subset \varrho$ and, since $\theta \not \subset M_{\sigma}$, we deduce that $\varrho \not \subset M_{\sigma}$. By the properties of $K_{\epsilon}$, there exists a face $\zeta$ of $\varrho$ such that $\zeta^{0} \subset \varrho \backslash M_{\epsilon} \subset \zeta$. Observe that since $\varrho \subset \epsilon$, we have

$$
\zeta^{0} \subset \varrho \backslash M_{\epsilon}=\varrho \backslash(M \cap \epsilon)=\varrho \backslash M \subset \theta \backslash M=\theta \backslash M_{\sigma} \subset \varrho \backslash M_{\sigma}=\varrho \backslash M_{\epsilon} \subset \zeta
$$

and so $\zeta^{0} \subset \theta \backslash M_{\sigma} \subset \zeta$, which proves the claim of 2.7/3.

Now, let $K_{1}$ be a triangulation of $X$ compatible with all the open faces of all the simplices in the triangulations $K_{\sigma}$ of all the $d$-dimensional simplices of $K_{0}$. Notice that, by 2.8 for all $\theta \in K_{1}$, either $\theta \subset M$ or there exists a face $\zeta$ of $\theta$ such that $\zeta^{0} \subset \theta \backslash M \subset \zeta$.

(2.74) We now study the general case. By 2.6, there exist a finite simplicial complex $K^{\prime}=\left\{s_{i}\right\}_{i=1}^{p}$ in $\mathbb{R}^{n}$ and a semialgebraic triangulation $\left(K^{\prime}, \Psi\right)$ of $X$ compatible with $\{M\}$. Proceeding as above with each simplex $s_{i}$, we get a triangulation $K_{i}$ of $s_{i}$ satisfying the conditions in the statement for $s_{i}$ and $\Psi^{-1}(M) \cap s_{i}$. Let $\mathcal{H}$ be the union of $\mathcal{F}$ and the family of all open faces of all simplices occurring in all triangulations $K_{i}$. By 2.6, there exists a semialgebraic triangulation $\left(K^{\prime \prime}, \Phi\right)$ of $X$ compatible with $\mathcal{H}$; by 2.8 this triangulation satisfies the conditions in the statement.

Corollary 2.9. Let $M \subset X \subset \mathbb{R}^{n}$ be semialgebraic sets such that $X$ is compact and $M$ is dense in $X$. Let $\mathcal{F}$ be a finite family of semialgebraic subsets of $X$ containing $M$. Then, there exists a semialgebraic triangulation $(K, \Phi)$ of $X$ compatible 
with $\mathcal{F}$ such that, for each simplex $\sigma \in K$ which is not a face of other simplex of the triangulation $(K, \Phi)$, either $\sigma \subset \Phi^{-1}(M)$ or there exists a proper face $\tau$ of $\sigma$ satisfying $\tau^{0} \subset \sigma \backslash \Phi^{-1}(M) \subset \tau$.

Proof. First, by 2.7, there exists a semialgebraic triangulation $(K, \Phi)$ of $X$ compatible with the family $\mathcal{F}$ such that for each simplex $\sigma \in K$ either $\sigma \subset \Phi^{-1}(M)$ or there exists a face $\tau$ of $\sigma$ satisfying $\tau^{0} \subset \sigma \backslash \Phi^{-1}(M) \subset \tau$. Let us check that if $\sigma \in K$ is not a face of other simplex of the triangulation $(K, \Phi)$ and $\sigma \not \subset \Phi^{-1}(M)$, then $\tau$ is a proper face of $\sigma$.

Indeed, let $\sigma_{1}, \ldots, \sigma_{s}$ be the collection of all simplices of $K$ different from $\sigma$. Since $\sigma$ is not a face of other simplex of the triangulation $(K, \Phi)$, we deduce that $\sigma^{0}=|K| \backslash \bigcup_{i=1}^{s} \sigma_{i}$, and so $\Phi\left(\sigma^{0}\right)$ is a nonempty open subset of $X$. Now, since $M$ is dense in $X$, the intersection $\Phi\left(\sigma^{0}\right) \cap M$ is nonempty. This implies, since $(K, \Phi)$ is compatible with $M$, that $\Phi\left(\sigma^{0}\right) \subset M$. This, together with the inclusion $\tau^{0} \subset \sigma \backslash \Phi^{-1}(M)$, implies that $\tau \neq \sigma$, that is, $\tau$ is a proper face of $\sigma$, as wanted.

\section{Maximal SPeCtra of Rings of Semialgebraic FunCtions}

The purpose of this section is to study the maximal spectra of rings of semialgebraic and bounded semialgebraic functions on a semialgebraic set $M \subset \mathbb{R}^{n}$. As we will see in this section both maximal spectra of $\mathcal{S}(M)$ and $\mathcal{S}^{*}(M)$ are Hausdorff compactifications of $M$ with nice properties, which are in fact homeomorphic. We refer the reader to $\mathrm{BCR}, \S 1, \S 7$ for more details about real or orderable fields and the real spectrum of a commutative ring with unity.

(3.1) Maximal spectra. We will denote by $\beta_{\mathrm{s}}^{\diamond} M$ the collection of all maximal ideals of $\mathcal{S}^{\diamond}(M)$. As usual, we consider in $\beta_{\mathrm{s}}^{\diamond} M$ the Zariski topology, having the family of sets $\mathcal{D}_{\beta_{\mathrm{s}}^{\diamond} M}(f)=\left\{\mathfrak{m} \in \beta_{\mathrm{s}}^{\diamond} M: f \notin \mathfrak{m}\right\}$, where $f \in \mathcal{S}^{\diamond}(M)$, as a basis of open sets. We will denote $\mathcal{Z}_{\beta_{\mathrm{s}}^{\diamond} M}(f)=\beta_{\mathrm{s}}^{\diamond} M \backslash \mathcal{D}_{\beta_{\mathrm{s}} M}(f)$.

[3.11) We recall that: for each maximal ideal $\mathfrak{m}$ of $\mathcal{S}^{\diamond}(M)$, the field $\mathcal{S}^{\diamond}(M) / \mathfrak{m}$ admits a unique ordering whose positive elements are the squares. Indeed, it is enough to check that modulo $\mathfrak{m}$ each function $f \in \mathcal{S}^{\diamond}(M)$ is either a square or the opposite of a square. Since $(f-|f|)(f+|f|)=f^{2}-|f|^{2}=0 \in \mathfrak{m}$, we have $f+\mathfrak{m}= \pm(|f|+\mathfrak{m})= \pm(\sqrt{|f|}+\mathfrak{m})^{2}$, where $\sqrt{|f|} \in \mathcal{S}^{\diamond}(M)$.

(3.12) Hence, the map $\mathfrak{m} \mapsto(\mathfrak{m}, \leq)$ defines a bijection between $\beta_{\mathrm{s}}^{\diamond} M$ and the real maximal spectrum of $\mathcal{S}^{\diamond}(M)$. Thus, in what follows we will denote both spectra by $\beta_{\mathrm{s}}^{\diamond M}$. Recall that a basis of the usual spectral topology for $\beta_{\mathrm{s}}^{\diamond} M$ is the family of sets

$$
\mathcal{U}_{\beta_{\mathrm{s}} M}\left(f_{1}, \ldots, f_{r}\right)=\left\{\mathfrak{m} \in \beta_{\mathrm{s}}^{\diamond} M: f_{i}+\mathfrak{m}>0 \text { in } \mathcal{S}^{\diamond}(M) / \mathfrak{m}\right\},
$$

for $f_{1}, \ldots, f_{r} \in \mathcal{S}^{\diamond}(M)$. As one can check straightforwardly, for each $f \in \mathcal{S}^{\diamond}(M)$,

$$
\mathcal{D}_{\beta_{\mathrm{s}}^{\diamond} M}(f)=\mathcal{U}_{\beta_{\mathrm{s}} M}(f) \cup \mathcal{U}_{\beta_{\mathrm{s}}^{\diamond} M}(-f) \quad \text { and } \quad \mathcal{U}_{\beta_{\mathrm{s}}^{\diamond} M}(f)=\mathcal{D}_{\beta_{\mathrm{s}}^{\diamond} M}(f+|f|),
$$

and so, as is well known, the spectral and the Zariski topologies of $\beta_{\mathrm{s}}^{\diamond} M$ coincide.

(3.13) At this point we also recall that $M$ (endowed with the Euclidean topology) can be embedded in $\beta_{\mathrm{s}}^{\diamond} M$ as a dense subspace via the embedding

$$
\phi: M \rightarrow \beta_{\mathrm{s}}^{\diamond} M, p \mapsto \mathfrak{m}_{p}^{\diamond},
$$

where $\mathfrak{m}_{p}^{\diamond}$ denotes the maximal ideal of all functions of $\mathcal{S}^{\diamond}(M)$ vanishing at $p$. Thus, we identify $M$ with $\phi(M)$ and this provides the equalities $D_{M}(f)=\mathcal{D}_{\beta_{\mathrm{s}}^{\diamond} M}(f) \cap M$ and $Z_{M}(f)=Z_{\beta_{s}^{\diamond} M}(f) \cap M$. 
The operator $\beta_{\mathrm{s}}^{\diamond}$ enjoys the expected functorial behaviour (see for instance [FG3, §6] for further details). In fact, given a semialgebraic map $\varphi: N \rightarrow M$ between semialgebraic sets $N \subset \mathbb{R}^{n}$ and $M \subset \mathbb{R}^{m}$, there exists a unique continuous extension $\beta_{\mathrm{s}}^{\diamond} \varphi: \beta_{\mathrm{s}}^{\diamond} N \rightarrow \beta_{\mathrm{s}}^{\diamond} M$ of $\varphi$.

(3.14) Moreover, in [FG3, 6.3-5] we prove that $\beta_{\mathrm{s}}^{\diamond}$ enjoys a natural behaviour with respect to the closed semialgebraic subsets of a given semialgebraic set. More precisely, let $C, C_{1}, C_{2}$ be closed semialgebraic subsets of $M$. Then,

(i) The space $\beta_{\mathrm{s}}^{\diamond} C$ is homeomorphic to $\mathrm{Cl}_{\beta_{\mathrm{s}}^{\diamond} M}(C) \subset \beta_{\mathrm{s}}^{\diamond} M$ via $\beta_{\mathrm{s}}^{\diamond} j: \beta_{\mathrm{s}}^{\diamond} C \rightarrow \beta_{\mathrm{s}}^{\diamond} M$, where $j: C \hookrightarrow M$ is the inclusion map.

(ii) $\mathrm{Cl}_{\beta_{\S}^{\diamond} M}\left(C_{1} \cap C_{2}\right)=\mathrm{Cl}_{\beta_{\S}^{\diamond} M}\left(C_{1}\right) \cap \mathrm{Cl}_{\beta_{\S}^{\diamond} M}\left(C_{2}\right)$.

(3.15) Concerning the connected components of the maximal spectrum of $\mathcal{S}^{\diamond}(M)$, the situation is the one we desire (see [FG3, 6.6]). Let $M_{1}, \ldots, M_{k}$ be the connected components of a semialgebraic set $M \subset \mathbb{R}^{n}$. Then, their closures $\mathrm{Cl}_{\beta_{\mathrm{s}} M}\left(M_{i}\right) \cong$ $\beta_{\mathrm{s}}^{\diamond} M_{i}$ are the connected components of $\beta_{\mathrm{s}}^{\diamond} M$. In particular, $\beta_{\mathrm{s}}^{\diamond} M$ has a finite number of connected components, and it is connected if and only if $M$ is connected too.

(3.16) On the other hand, by [BCR, 7.1.25(ii)], $\beta_{\mathrm{s}}^{\diamond} M$ is a compact, Hausdorff space and, by 3.113, it contains $M$ as a dense subspace, that is, $\beta_{\mathrm{s}}^{\diamond} M$ is a Hausdorff compactification of $M$. Now let us see that, as it happens for rings of continuous functions (see [GJ, §7]), the respective maximal spectra $\beta_{\mathrm{s}} M$ and $\beta_{\mathrm{s}}^{*} M$ of $\mathcal{S}(M)$ and $\mathcal{S}^{*}(M)$ are homeomorphic. We recall at this point that $\mathcal{S}^{\diamond}(M)$ is a Gelfand ring (see [FG2, 3.1(iii)]). Hence, a natural map from $\beta_{\mathrm{s}} M$ to $\beta_{\mathrm{s}}^{*} M$ associates to each maximal ideal $\mathfrak{m}$ of $\mathcal{S}(M)$ the unique maximal ideal $\mathfrak{m}^{*}$ of $\mathcal{S}^{*}(M)$ that contains the prime ideal $\mathfrak{m} \cap \mathcal{S}^{*}(M)$. Before proving that this map is a homeomorphism, we need some preliminary results.

Definitions 3.2. Let $M \subset \mathbb{R}^{n}$ be a semialgebraic set. For every $f \in \mathcal{S}(M)$ and $\varepsilon>0$ we denote

$$
B_{\varepsilon}(f)=f^{-1}([-\varepsilon, \varepsilon])=Z_{M}(\varepsilon-|f|-|\varepsilon-| f||)=B_{\varepsilon}(|f|) .
$$

Let us fix in what follows an ideal $\mathfrak{a}$ of $\mathcal{S}(M)$, and define

$$
\mathfrak{a}^{*}=\left\{f \in \mathcal{S}^{*}(M): \forall \varepsilon>0 \exists g \in \mathfrak{a} \text { with } Z_{M}(g) \subset B_{\varepsilon}(f)\right\},
$$

which, as one can check straightforwardly, is a radical ideal which satisfies the following convexity condition: given $h \in \mathcal{S}^{*}(M)$ and $f \in \mathfrak{a}^{*}$ such that $0 \leq h(x) \leq$ $f(x)$ for each $x \in M$, then $h \in \mathfrak{a}^{*}$.

We will next prove some preliminary results.

Lemma 3.3. Let $M \subset \mathbb{R}^{n}$ be a semialgebraic set and let $\mathfrak{p}$ be a prime ideal of $\mathcal{S}(M)$ and $\mathfrak{m}$ the unique maximal ideal of $\mathcal{S}(M)$ containing $\mathfrak{p}$. Then, $\mathfrak{p}^{*}=\mathfrak{m}^{*}$ is the unique maximal ideal in $\mathcal{S}^{*}(M)$ containing $\mathfrak{p} \cap \mathcal{S}^{*}(M)$.

Proof. First, let us prove that $\mathfrak{p}^{*}=\mathfrak{m}^{*}$. The inclusion $\mathfrak{p}^{*} \subset \mathfrak{m}^{*}$ is clear, and let us check that in fact it is an equality. Suppose by way of contradiction that there exists $f \in \mathfrak{m}^{*} \backslash \mathfrak{p}^{*}$. Since $f \notin \mathfrak{p}^{*}$ there exists $\varepsilon>0$ such that for all $g \in \mathcal{S}(M)$ with $Z_{M}(g) \subset B_{\varepsilon}(f)$, we have $g \notin \mathfrak{p}$. In particular, the function $g_{1}=\varepsilon-|f|-|\varepsilon-| f||$ satisfies $B_{\varepsilon}(f)=Z_{M}\left(g_{1}\right)$, and so $g_{1} \in \mathcal{S}(M) \backslash \mathfrak{p}$. On the other hand, since $f \in \mathfrak{m}^{*}$, there exists $g_{2} \in \mathfrak{m}$ such that $Z_{M}\left(g_{2}\right) \subset B_{\varepsilon / 2}(f)$. Next, by 2.2. there exists $h \in$ 
$\mathcal{S}(M)$ such that $Z_{M}(h)=\{x \in M:|f(x)| \geq \varepsilon\}$. Since $g_{1} h=0 \in \mathfrak{p}$ and $g_{1} \notin \mathfrak{p}$, we deduce that $h \in \mathfrak{p} \subset \mathfrak{m}$ and so $g_{2}^{2}+h^{2} \in \mathfrak{m}$. However,

$$
Z_{M}\left(g_{2}^{2}+h^{2}\right) \subset B_{\varepsilon / 2}(f) \cap\{x \in M:|f(x)| \geq \varepsilon\}=\varnothing,
$$

or equivalently, $g_{2}^{2}+h^{2} \in \mathfrak{m}$ is a unit of $\mathcal{S}(M)$, a contradiction. Hence, $\mathfrak{p}^{*}=\mathfrak{m}^{*}$.

Thus, to prove the second part of the statement, we may assume that $\mathfrak{p}=\mathfrak{m}$ is a maximal ideal of $\mathcal{S}(M)$. We already know that $\mathfrak{m}^{*}$ is an ideal of $\mathcal{S}^{*}(M)$. Before proving that $\mathfrak{m}^{*}$ is maximal we show that $\mathfrak{m} \cap \mathcal{S}^{*}(M) \subset \mathfrak{m}^{*}$. Let $f \in \mathfrak{m} \cap \mathcal{S}^{*}(M)$; given $\varepsilon>0$ there exists, by 2.2. $g \in \mathcal{S}(M)$ such that $B_{\varepsilon}(f)=Z_{M}(g)$, and it suffices to check that $g \in \mathfrak{m}$. By 2.2, there exists $h \in \mathcal{S}(M)$ such that

$$
\{x \in M:|f(x)| \geq \varepsilon\}=Z_{M}(h) .
$$

Then, $g h \equiv 0 \in \mathfrak{m}$, and so it is enough to prove that $h \notin \mathfrak{m}$, or equivalently, $f^{2}+h^{2} \notin \mathfrak{m}$, but this is obvious because $Z_{M}\left(f^{2}+h^{2}\right)=\varnothing$.

To finish let us prove that $\mathfrak{m}^{*}$ is a maximal ideal of $\mathcal{S}^{*}(M)$. Given $f \in \mathcal{S}^{*}(M) \backslash \mathfrak{m}^{*}$ we must show that $1 \in f \mathcal{S}^{*}(M)+\mathfrak{m}^{*}$. Since $f^{2} \notin \mathfrak{m}^{*}$ there exists $0<\varepsilon<1 / 2$ and $g \in \mathcal{S}(M) \backslash \mathfrak{m}$ such that $Z_{M}(g)=B_{\varepsilon}\left(f^{2}\right)$. Moreover, since $\mathfrak{m}$ is a maximal ideal and $g \notin \mathfrak{m}$, there exist $h \in \mathcal{S}(M)$ and $b \in \mathfrak{m}$ such that $1=g h+b$. Notice that $c=|b| /\left(1+b^{2}\right) \in \mathfrak{m} \cap \mathcal{S}^{*}(M) \subset \mathfrak{m}^{*}$ and $B_{\varepsilon}\left(f^{2}\right) \cap B_{\varepsilon}(c)=Z_{M}(g) \cap B_{\varepsilon}(c)=\varnothing$. Thus, $\varepsilon \leq f^{2}(x)+c(x)$ for each $x \in M$; hence, $d=1 /\left(f^{2}+c\right) \in \mathcal{S}^{*}(M)$ and $1=d\left(f^{2}+c\right)=f^{2} d+c d \in f \mathcal{S}^{*}(M)+\mathfrak{m}^{*}$, as wanted.

Lemma 3.4. Let $M \subset \mathbb{R}^{n}$ be a semialgebraic set and let $\mathfrak{m}$ be a maximal ideal of $\mathcal{S}(M)$ and $f \in \mathcal{S}(M)$. Then, the following conditions are equivalent:

(i) The function $f \in \mathfrak{m}$.

(ii) $Z_{M}(f) \cap B_{\varepsilon}(b) \neq \varnothing$ for each $\varepsilon>0$ and each $b \in \mathfrak{m}^{*}$.

Proof. Suppose first that $f \in \mathfrak{m}$, and let $\varepsilon>0$ and $b \in \mathfrak{m}^{*}$. Then, there exists $g \in \mathfrak{m}$ such that $Z_{M}(g) \subset B_{\varepsilon}(b)$. Thus,

$$
\varnothing \neq Z_{M}\left(f^{2}+g^{2}\right)=Z_{M}(f) \cap Z_{M}(g) \subset Z_{M}(f) \cap B_{\varepsilon}(b),
$$

because $f^{2}+g^{2} \in \mathfrak{m}$.

Conversely, let $f \in \mathcal{S}(M)$ be a semialgebraic function satisfying (ii). We will prove:

(3.41) $Z_{M}(f) \cap Z_{M}(g) \neq \varnothing \quad$ for all $g \in \mathfrak{m}$.

Once this is done, suppose, by way of contradiction, that $f \notin \mathfrak{m}$. Then, there exists $h \in \mathcal{S}(M)$ with $g=h f-1 \in \mathfrak{m}$, and so $Z_{M}(f) \cap Z_{M}(g)=\varnothing$, which contradicts $3.4[1$.

Next, assume, again by way of contradiction, that 3.4[1] does not hold. Then, there exists $g \in \mathfrak{m}$ such that $f$ and $g$ have no common zero. Therefore, the semialgebraic function $h=g^{2} /\left(f^{2}+g^{2}\right) \in \mathcal{S}^{*}(M)$ satisfies $\left.h\right|_{Z_{M}(f)} \equiv 1$ and $\left.h\right|_{Z_{M}(g)} \equiv 0$. In particular, $h \in \mathcal{S}^{*}(M)$ and for every $\varepsilon>0, Z_{M}(g)=Z_{M}(h) \subset B_{\varepsilon}(h)$; hence, we have $h \in \mathfrak{m}^{*}$. Since $f$ fulfills condition (ii) in the statement, there exists $x \in Z_{M}(f) \cap B_{1 / 2}(h)$, and so $1=h(x) \leq 1 / 2$, a contradiction.

Theorem 3.5. Let $M \subset \mathbb{R}^{n}$ be a semialgebraic set. Then, the map

$$
\Phi: \beta_{\mathrm{s}} M \rightarrow \beta_{\mathrm{s}}^{*} M, \mathfrak{m} \mapsto \mathfrak{m}^{*},
$$

which maps each maximal ideal $\mathfrak{m}$ of $\mathcal{S}(M)$ to the unique maximal ideal $\mathfrak{m}^{*}$ of $\mathcal{S}^{*}(M)$ that contains $\mathfrak{m} \cap \mathcal{S}^{*}(M)$, is a homeomorphism. Moreover, $\Phi\left(\mathfrak{m}_{p}\right)=\mathfrak{m}_{p}^{*}$ for all $p \in M$. 
Proof. First, we prove that the map $\Phi$ is continuous, proper and surjective. Indeed, the map $(\cdot)^{*}: \operatorname{Spec}_{\mathrm{s}}(M) \rightarrow \beta_{\mathrm{s}}^{*} M, \mathfrak{p} \rightarrow \mathfrak{p}^{*}$ is the composition $\mathrm{r}_{M} \circ j_{1}$ of the continuous map $j_{1}: \operatorname{Spec}_{\mathrm{s}}(M) \rightarrow \operatorname{Spec}_{\mathrm{s}}^{*}(M), \mathfrak{p} \rightarrow \mathfrak{p} \cap \mathcal{S}^{*}(M)$ with the (continuous) retraction $\mathrm{r}_{M}: \operatorname{Spec}_{\mathrm{s}}^{*}(M) \rightarrow \beta_{\mathrm{s}}^{*} M$ which maps each prime ideal of $\mathcal{S}^{*}(M)$ onto the unique maximal ideal containing it (see [MO, 1.2]). Hence, the map $(\cdot)^{*}$ is continuous. Thus, if $j_{2}: \beta_{\mathrm{s}} M \hookrightarrow \operatorname{Spec}_{\mathrm{s}}(M)$ is the inclusion map, then the composition

$$
\Phi=\mathrm{r}_{M} \circ j_{1} \circ j_{2}=(\cdot)^{*} \circ j_{2}: \beta_{\mathrm{s}} M \rightarrow \beta_{\mathrm{s}}^{*} M
$$

is also continuous. Moreover, since $\mathfrak{m}_{p} \cap \mathcal{S}^{*}(M)=\mathfrak{m}_{p}^{*}$ for each $p \in M$, we deduce that $\Phi\left(\mathfrak{m}_{p}\right)=\mathfrak{m}_{p}^{*}$ for all $p \in M$. Thus, since $\beta_{\mathbf{s}} M$ is compact, the set im $\Phi$, which contains $M$, is closed in the Hausdorff space $\beta_{\mathrm{s}}^{*} M$. Hence, $\operatorname{im} \Phi=\mathrm{Cl}_{\beta_{\mathrm{s}}^{*} M}(M)=$ $\beta_{\mathrm{s}}^{*} M$, and so $\Phi$ is surjective. Therefore, $\Phi: \beta_{\mathrm{s}} M \rightarrow \beta_{\mathrm{s}}^{*} M$ is continuous, proper and surjective, and it only remains to prove its injectivity.

Indeed, let $\mathfrak{m}_{1}$ and $\mathfrak{m}_{2}$ be distinct maximal ideals of $\mathcal{S}(M)$, and let $f \in \mathfrak{m}_{1} \backslash \mathfrak{m}_{2}$. Then, by [3.4, there exist $\varepsilon>0$ and $b \in \mathfrak{m}_{2}^{*}$ such that $Z_{M}(f) \cap B_{\varepsilon}(b)=\varnothing$. Since $f \in \mathfrak{m}_{1}$, necessarily $b \notin \mathfrak{m}_{1}^{*}$ (again use 3.4 ). Thus, $\mathfrak{m}_{1}^{*} \neq \mathfrak{m}_{2}^{*}$, and we are done.

Remarks 3.6. (i) Notice that the inverse homeomorphism

$$
\Phi^{-1}: \beta_{\mathrm{s}}^{*} M \rightarrow \beta_{\mathrm{s}} M, \mathfrak{m}^{*} \mapsto \mathfrak{m}
$$

is defined by $\mathfrak{m}=\left\{f \in \mathcal{S}(M): Z_{M}(f) \cap B_{\varepsilon}(b) \neq \varnothing \quad \forall \varepsilon>0 \& \forall b \in \mathfrak{m}^{*}\right\}$ (see 3.4).

(ii) As a consequence of 3.5 it is not an abuse of notation to denote $\mathfrak{m}^{*}$ for every maximal ideal of $\mathcal{S}^{*}(M)$. Moreover, $\mathfrak{m}$ will denote the unique maximal ideal of $\mathcal{S}(M)$ such that $\mathfrak{m} \cap \mathcal{S}^{*}(M) \subset \mathfrak{m}^{*}$.

(iii) Observe that the inclusion map $\mathbb{R} \hookrightarrow \mathcal{S}^{*}(M) / \mathfrak{m}^{*}, r \mapsto r+\mathfrak{m}^{*}$, is an (injective) homomorphism of ordered fields; in fact, it is an isomorphism, because $\mathcal{S}^{*}(M) / \mathfrak{m}^{*}$ is an archimedean extension of $\mathbb{R}$. Thus, since $\mathbb{R}$ admits a unique automorphism, there is no ambiguity to refer to $f+\mathfrak{m}^{*} \in \mathbb{R}$ as a real number for every $f \in \mathcal{S}^{*}(M)$. In particular, for each $p \in M$ the isomorphism $\mathcal{S}^{*}(M) / \mathfrak{m}_{p}^{*} \cong \mathbb{R}$ identifies $f+\mathfrak{m}_{p}^{*}$ with $f(p)$. Therefore, each bounded semialgebraic function $f: M \rightarrow \mathbb{R}$ defines a natural extension $\widehat{f}: \beta_{\mathrm{s}}^{*} M \rightarrow \mathbb{R}, \mathfrak{m}^{*} \rightarrow f+\mathfrak{m}^{*}$, which is continuous because $\widehat{f}^{-1}((a, b))=\mathcal{U}_{\beta_{\mathrm{s}}^{*} M}(f-a, b-f)$ for every pair of real numbers $a<b$. Of course, $\widehat{f}$ is the unique continuous extension of $f$ to $\beta_{\mathrm{s}}^{*} M$ because $M$ is dense in $\beta_{\mathrm{s}}^{*} M$.

In contrast to what happens in dealing with ideals of polynomial rings, the zeroset of a prime ideal $\mathfrak{p}$ of $\mathcal{S}^{\diamond}(M)$ provides no substantial information about $\mathfrak{p}$, because it is either a point or the empty set (see for instance [FG1, 2.3]). An ideal $\mathfrak{a}$ of $\mathcal{S}^{\diamond}(M)$ is said to be fixed if all functions in $\mathfrak{a}$ vanish simultaneously at some point of $M$. Otherwise, the ideal $\mathfrak{a}$ is free. The fixed maximal ideals of the $\operatorname{ring} \mathcal{S}^{\diamond}(M)$ are those of the form $\mathfrak{m}_{p}^{\diamond}$ where $p \in M$. As we have already commented, $\mathfrak{m}_{p} \cap \mathcal{S}^{*}(M)=\mathfrak{m}_{p}^{*}$ for each point $p \in M$. In fact, the equality $\mathfrak{m} \cap \mathcal{S}^{*}(M)=\mathfrak{m}^{*}$ characterizes the fixed maximal ideals of $\mathcal{S}^{\diamond}(M)$. Namely, if ht(a) denotes the height of an ideal $\mathfrak{a}$, we have:

Proposition 3.7. Let $M \subset \mathbb{R}^{n}$ be a semialgebraic set and let $\mathfrak{m}$ be a maximal ideal of $\mathcal{S}(M)$. Then, the following assertions are equivalent:

(i) $\mathfrak{m}^{*}$ is a fixed ideal.

(ii) $\mathfrak{m} \cap \mathcal{S}^{*}(M)=\mathfrak{m}^{*}$. 
(iii) $\mathfrak{m}$ is a fixed ideal.

(iv) $\operatorname{ht}(\mathfrak{m})=\operatorname{ht}\left(\mathfrak{m}^{*}\right)$.

Before proving 3.7 we need some preliminaries.

Proposition 3.8. Let $M \subset \mathbb{R}^{n}$ be a semialgebraic set and $f \in \mathcal{S}^{\diamond}(M)$.

(i) If the zeroset $Z_{M}(f)$ is not compact, then $f$ lies in some free ideal $\mathfrak{a}$ of $\mathcal{S}^{\diamond}(M)$.

(ii) If $\mathcal{S}^{\diamond}(M)=\mathcal{S}(M)$, the converse of (i) is true.

Proof. (i) Since $Z_{M}(f)$ is not compact, there exists a family $\left\{W_{i}\right\}_{i \in I}$ of open semialgebraic subsets of $\mathbb{R}^{n}$ which covers $Z_{M}(f)$ and admits no finite subcovering. For each index $i \in I$, let $g_{i} \in \mathcal{S}^{*}\left(\mathbb{R}^{n}\right)$ such that $Z_{\mathbb{R}^{n}}\left(g_{i}\right)=\mathbb{R}^{n} \backslash W_{i}$. Let us show that the ideal $\mathfrak{a}$ of $\mathcal{S}^{\diamond}(M)$ generated by $f$ and the restrictions $f_{i}=\left.g_{i}\right|_{M}$ is a free ideal. In case $\mathfrak{a}=\mathcal{S}^{\diamond}(M)$ we have an equality $1=g f+\sum_{j \in J} f_{j} h_{j}$ for some finite subset $J$ of $I$ and some functions $g, h_{j} \in \mathcal{S}^{\diamond}(M)$. Since the finite family $\left\{W_{j}\right\}_{j \in J}$ does not cover $Z_{M}(f)$ there exists a point $x \in Z_{M}(f) \backslash \bigcup_{j \in J} W_{j}$, which contradicts the equality above. Thus, $\mathfrak{a}$ is a proper ideal of $\mathcal{S}^{\diamond}(M)$, and we now check that it is free. In fact, $Z_{M}(f) \subset \bigcup_{i \in I} W_{i}$ or, equivalently,

$$
\bigcap_{h \in \mathfrak{a}} Z_{M}(h)=Z_{M}(f) \cap \bigcap_{i \in I} Z_{M}\left(f_{i}\right)=Z_{M}(f) \cap \bigcap_{i \in I}\left(\mathbb{R}^{n} \backslash W_{i}\right)=\varnothing,
$$

and so $\mathfrak{a}$ is a free ideal.

To prove (ii), assume that $f \in \mathfrak{a}$ for some free ideal $\mathfrak{a}$ of $\mathcal{S}(M)$. Then, the intersection $\bigcap_{g \in \mathfrak{a}} Z_{M}(g)=\varnothing$, that is, the family $\left\{D_{M}(g): g \in \mathfrak{a}\right\}$, is an open covering of $Z_{M}(f)$. Notice that the finite unions $D_{M}\left(g_{1}\right) \cup \cdots \cup D_{M}\left(g_{r}\right)$ do not cover $Z_{M}(f)$, and so this last is not compact. Otherwise, if

$$
Z_{M}(f) \subset D_{M}\left(g_{1}\right) \cup \cdots \cup D_{M}\left(g_{r}\right)
$$

for some $g_{1}, \ldots, g_{r} \in \mathfrak{a}$, the intersection $Z_{M}(f) \cap Z_{M}\left(g_{1}\right) \cap \cdots \cap Z_{M}\left(g_{r}\right)=\varnothing$, and this implies that $f^{2}+g_{1}^{2}+\cdots+g_{r}^{2} \in \mathfrak{a}$ would be a unit in $\mathcal{S}(M)$, a contradiction.

Remark 3.9. Note that 3.8(ii) is not true for $\mathcal{S}^{*}(M)$ if $M$ is not compact. Indeed, we may assume, by 2.1. that $M$ is bounded, and so there is a point $p \in \mathrm{Cl}_{\mathbb{R}^{n}}(M) \backslash M$. Consider the bounded semialgebraic function $f: M \rightarrow \mathbb{R}, x \rightarrow\|x-p\|$ whose zeroset is empty, hence, compact, and the ideal generated by $f$ in $\mathcal{S}^{*}(M)$ is free. This also proves that if $M$ is not compact, then $\mathcal{S}^{*}(M)$ has free maximal ideals. Of course, if $M$ is a compact semialgebraic set there is nothing to discuss because $\mathcal{S}(M)=\mathcal{S}^{*}(M)$.

Now, we are ready to prove 3.7

Proof of Proposition 3.7. The equivalence (i) $\Longleftrightarrow$ (iii) is clear, and the one of (ii) and (iv) follows from the fact that $\mathcal{S}(M)=\mathcal{S}^{*}(M)_{\mathcal{W}(M)}$ is the localization of $\mathcal{S}^{*}(M)$ at the multiplicative subset $\mathcal{W}(M)$ of those bounded semialgebraic functions on $M$ with empty zeroset. Thus, it suffices to check the implications (i) $\Longrightarrow$ (ii) $\Longrightarrow$ (iii).

Indeed, if $\mathfrak{m}^{*}=\mathfrak{m}_{p}^{*}$ for some $p \in M$, then $\mathfrak{m}_{p} \cap \mathcal{S}^{*}(M)=\mathfrak{m}_{p}^{*}$. Next, let $\mathfrak{m}$ be a maximal ideal of $\mathcal{S}(M)$ such that $\mathfrak{m} \cap \mathcal{S}^{*}(M)=\mathfrak{m}^{*}$. By 2.1. we may assume that $M$ is bounded and we consider the compact semialgebraic set $X=\mathrm{Cl}_{\mathbb{R}^{n}}(M)$. We have the following sequence of ring monomorphisms

$$
\mathcal{S}(X)=\mathcal{S}^{*}(X) \hookrightarrow \mathcal{S}^{*}(M) \hookrightarrow \mathcal{S}(M),
$$


where the first one is defined as the restriction to $M$, while the second one is the inclusion. Let us see that $\mathfrak{n}=\mathfrak{m}^{*} \cap \mathcal{S}(X)=\mathfrak{m} \cap \mathcal{S}^{*}(M) \cap \mathcal{S}(X)=\mathfrak{m} \cap \mathcal{S}(X)$ is a maximal ideal of $\mathcal{S}(X)$. Consider the following composition of homomorphisms:

$$
\psi: \mathcal{S}(X) \hookrightarrow \mathcal{S}^{*}(M) \rightarrow \mathcal{S}^{*}(M) / \mathfrak{m}^{*} \cong \mathbb{R} .
$$

Since $\mathfrak{n}=\operatorname{ker} \psi$, we get $\mathbb{R} \hookrightarrow \mathcal{S}(X) / \mathfrak{n} \rightarrow \mathcal{S}^{*}(M) / \mathfrak{m}^{*} \cong \mathbb{R}$, and so $\mathcal{S}(X) / \mathfrak{n} \cong \mathbb{R}$; that is, $\mathfrak{n}$ is a maximal ideal of $\mathcal{S}(X)$. Next, by the compactness of $X$ there exists, by 3.8, a point $p \in X$ such that $\mathfrak{n}=\mathfrak{n}_{p}=\{f \in \mathcal{S}(X): f(p)=0\}$. The function $g(x)=\|x-p\| \in \mathfrak{n}=\mathfrak{m} \cap \mathcal{S}(X) \subset \mathfrak{m}$. Since $\mathfrak{m}$ is an ideal of $\mathcal{S}(M)$, the point $p \in M$ (otherwise $g$ would be a unit of $\mathcal{S}(M)$ ). Thus, $\mathfrak{m} \subset \mathfrak{m}_{p}$, because if not, there exists $h \in \mathfrak{m}$ such that $h(p) \neq 0$; hence the zeroset of $g^{2}+h^{2} \in \mathfrak{m}$ is empty, a contradiction. Therefore, $\mathfrak{m}$ being a maximal ideal, we conclude that $\mathfrak{m}=\mathfrak{m}_{p}$ is a fixed ideal, as wanted.

Corollary 3.10. Let $M \subset \mathbb{R}^{n}$ be a semialgebraic set. Then, the following assertions are equivalent:

(i) $M$ is compact.

(ii) Each maximal ideal of $\mathcal{S}(M)$ is fixed.

(iii) Each maximal ideal of $\mathcal{S}^{*}(M)$ is fixed.

(iv) $\mathcal{S}(M)=\mathcal{S}^{*}(M)$.

Proof. The equivalence of (i) and (ii) follows from 3.8 and the one of (ii) and (iii) follows from 3.7. Finally, we check that the rings $\mathcal{S}(M)$ and $\mathcal{S}^{*}(M)$ coincide if and only if $M$ is compact. The equality $\mathcal{S}(M)=\mathcal{S}^{*}(M)$ is obvious if $M$ is compact. Conversely, if $\mathcal{S}(M)=\mathcal{S}^{*}(M)$, then $\mathfrak{m} \cap \mathcal{S}^{*}(M)=\mathfrak{m}^{*}$ for each maximal ideal $\mathfrak{m}$ of $\mathcal{S}(M)$. Thus, by 3.7 all maximal ideals of $\mathcal{S}(M)$ are fixed and, by the equivalence of (i) and (ii), $M$ is compact.

We have shown in 3.5 that the maximal spectra of $\mathcal{S}(M)$ and $\mathcal{S}^{*}(M)$ are homeomorphic. However, there exists a difference in dealing with them. While all quotients $\mathcal{S}^{*}(M) / \mathfrak{m}^{*}$ are (isomorphic to) $\mathbb{R}$, for the maximal ideals $\mathfrak{m}$ of the ring $\mathcal{S}(M)$ this is true if and only if $\mathfrak{m}$ is a fixed maximal ideal. Namely,

Corollary 3.11. Let $M \subset \mathbb{R}^{n}$ be a semialgebraic set and let $\mathfrak{m}$ be a maximal ideal of $\mathcal{S}(M)$. Then, $\mathcal{S}(M) / \mathfrak{m}$ is (isomorphic to) $\mathbb{R}$ if and only if $\mathfrak{m}$ is a fixed maximal ideal.

Proof. Note that $\mathcal{S}(M) / \mathfrak{m}_{p} \cong \mathbb{R}$ for every fixed maximal ideal $\mathfrak{m}_{p}$ of $\mathcal{S}(M)$, since $\mathfrak{m}_{p}$ is the kernel of the epimorphism evaluation at $p$. For the converse, consider the homomorphisms $\mathcal{S}^{*}(M) \hookrightarrow \mathcal{S}(M) \rightarrow \mathcal{S}(M) / \mathfrak{m} \cong \mathbb{R}$. The composition is surjective and its kernel is $\mathfrak{m} \cap \mathcal{S}^{*}(M)$, which is henceforth a maximal ideal of $\mathcal{S}^{*}(M)$. Thus, $\mathfrak{m} \cap \mathcal{S}^{*}(M)=\mathfrak{m}^{*}$ is, by 3.7, a fixed ideal of $\mathcal{S}^{*}(M)$. We conclude, again by 3.7 that $\mathfrak{m}$ is a fixed ideal of $\mathcal{S}(M)$.

\section{Semialgebraic Stone-Č́ech COmpactification}

The classical Stone-Čech compactification is a crucial tool for the study of rings of continuous functions. It is characterized as the "smallest" Hausdorff compactification to which all bounded $\mathbb{R}$-valued continuous functions extend continuously. This suggests that we make a similar construction in the semialgebraic setting. To illustrate the used strategy we begin by constructing a compactification to which a finite family of bounded semialgebraic functions can be extended. In this case, 
we can force the compactification to be moreover semialgebraic. As we will see later the semialgebraicity of a compactification to which all bounded semialgebraic functions extend is kept just for very particular situations.

Proposition 4.1. Let $M \subset \mathbb{R}^{n}$ be a semialgebraic set and let $f_{1}, \ldots, f_{r} \in \mathcal{S}^{*}(M)$. Then, there exists a compact semialgebraic set $X \subset \mathbb{R}^{n+r}$ and a semialgebraic embedding $j: M \hookrightarrow X$ such that $(X, j)$ is a compactification of $M$, and for each $f_{i}$ there exists $F_{i} \in \mathcal{S}(X)$ such that $F_{i} \circ j=f_{i}$. In fact, if $M$ is bounded, we can take $X=\mathrm{Cl}_{\mathbb{R}^{n+r}}\left(\operatorname{graph}\left(f_{1}, \ldots, f_{r}\right)\right)$.

Proof. First, we may assume, by 2.1, that $M$ is bounded. Consider the graph $\Gamma$ of the semialgebraic map $\varphi: M \rightarrow \mathbb{R}^{r}, x \mapsto\left(f_{1}(x), \ldots, f_{r}(x)\right)$. Let $X$ be the closure in $\mathbb{R}^{n+r}$ of $\Gamma$. Since $M$ and the functions $f_{1}, \ldots, f_{r}$ are bounded, the same holds true for $\Gamma$; hence, $X$ is compact. Let $j: M \rightarrow \Gamma \hookrightarrow X, x \mapsto\left(x, f_{1}(x), \ldots, f_{r}(x)\right)$ and, for $1 \leq i \leq r$, let $F_{i}=\left.\pi_{n+i}\right|_{X}$ be the restriction to $X$ of the projection $\pi_{n+i}: \mathbb{R}^{n+r} \rightarrow \mathbb{R},\left(x_{1}, \ldots, x_{n+r}\right) \rightarrow x_{n+i}$. Then, the pair $(X, j)$ and the functions $F_{i}$ satisfy the required conditions.

Once this is done, we attack the problem of extending simultaneously all bounded semialgebraic functions on a semialgebraic set.

Definitions 4.2. (i) Let $M \subset \mathbb{R}^{n}$ be a semialgebraic set. A compactification $(X, j)$ of $M$ is semialgebraically complete if for each $f \in \mathcal{S}^{*}(M)$ there exists a continuous function $F: X \rightarrow \mathbb{R}$ such that $f=F \circ j$.

(ii) Given two compactifications $\left(X_{1}, j_{1}\right)$ and $\left(X_{2}, j_{2}\right)$ of a semialgebraic set $M \subset$ $\mathbb{R}^{n}$, we say that $\left(X_{2}, j_{2}\right)$ dominates $\left(X_{1}, j_{1}\right)$, and we write $\left(X_{1}, j_{1}\right) \preccurlyeq\left(X_{2}, j_{2}\right)$ if there exists a continuous surjective map $\rho: X_{2} \rightarrow X_{1}$ such that $\rho \circ j_{2}=j_{1}$. Note that since $j_{i}(M)$ is dense in $X_{i}$ for $i=1,2$, the map $\rho$ is unique with such property.

The domination relation $\preccurlyeq$ is an order relation (up to homeomorphism compatible with the embeddings) in the set of all compactifications of $M$, and we look for the smallest one among those being semialgebraically complete. Before that we need some preliminaries.

Lemma 4.3. Let $\left(X_{1}, j_{1}\right)$ and $\left(X_{2}, j_{2}\right)$ be two compactifications of a semialgebraic set $M \subset \mathbb{R}^{n}$ such that $\left(X_{1}, j_{1}\right) \preccurlyeq\left(X_{2}, j_{2}\right)$. Let $\rho: X_{2} \rightarrow X_{1}$ be the unique continuous and surjective map such that $\rho \circ j_{2}=j_{1}$.

(i) Suppose that $X_{2}$ is Hausdorff. Then, $\rho^{-1}\left(X_{1} \backslash j_{1}(M)\right)=X_{2} \backslash j_{2}(M)$. In particular, $\rho\left(X_{2} \backslash j_{2}(M)\right)=X_{1} \backslash j_{1}(M)$.

(ii) Suppose that $\left(X_{2}, j_{2}\right) \preccurlyeq\left(X_{1}, j_{1}\right)$. Then, $\rho$ is a homeomorphism.

(iii) Let $f: X_{1} \rightarrow \mathbb{R}$ be a continuous function. Then, $f \circ \rho: X_{2} \rightarrow \mathbb{R}$ is the unique continuous function such that $f \circ j_{1}=(f \circ \rho) \circ j_{2}$.

Proof. Statement (i) alone requires some comments. Indeed, let us first see that $X_{2} \backslash j_{2}(M) \subset \rho^{-1}\left(X_{1} \backslash j_{1}(M)\right)$. Let $x_{2} \in X_{2} \backslash j_{2}(M)$. Since $j_{2}(M)$ is dense in $X_{2}$, there exists a net $\left\{s_{d}, D, \leq\right\}$ in $M$ such that the net $\left\{j_{2}\left(s_{d}\right), D, \leq\right\}$ converges to $x_{2}$. By continuity, the net $\left\{j_{1}\left(s_{d}\right)=\left(\rho \circ j_{2}\right)\left(s_{d}\right), D, \leq\right\}$ converges to $\rho\left(x_{2}\right)$. If this point occurs in $j_{1}(M)$, then the net $\left\{s_{d}, D, \leq\right\}$ converges to a point $y \in M$. Since $X_{2}$ is Hausdorff and the points $x_{2}$ and $j_{2}(y)$ are limits of the net $\left\{j_{2}\left(s_{d}\right), D, \leq\right\}$, we conclude that $x_{2}=j_{2}(y) \in j_{2}(M)$, a contradiction. Conversely, suppose there exists $x_{2} \in \rho^{-1}\left(X_{1} \backslash j_{1}(M)\right) \cap j_{2}(M)$. Then, $\rho\left(x_{2}\right) \notin j_{1}(M)$, but $x_{2}=j_{2}(y)$ for some $y \in M$. This implies $\rho\left(x_{2}\right)=\rho\left(j_{2}(y)\right)=j_{1}(y) \in j_{1}(M)$, a contradiction. 
Finally, since $\rho$ is surjective and $\rho^{-1}\left(X_{1} \backslash j_{1}(M)\right)=X_{2} \backslash j_{2}(M)$, we conclude that $\rho\left(X_{2} \backslash j_{2}(M)\right)=X_{1} \backslash j_{1}(M)$.

In the rest of this section, all the involved compactifications will be Hausdorff. To construct the smallest semialgebraically complete compactification of a semialgebraic set we imitate, adapted to the semialgebraic context, the classical method to construct the Stone-Cech compactification of a completely regular topological space.

(4.4) Semialgebraic Stone-Čech compactification. Let $M \subset \mathbb{R}^{n}$ be a semialgebraic set. For each semialgebraic function $f \in \mathcal{S}^{*}(M)$ consider the bounded interval $I_{f}=\left[\inf _{M}(f), \sup _{M}(f)\right] \subsetneq \mathbb{R}$, and let $\prod_{f \in \mathcal{S}^{*}(M)} I_{f}$ which, endowed with the product topology, is a compact and Hausdorff topological space.

(4.4.1) The map $\varphi: M \rightarrow \prod_{f \in \mathcal{S}^{*}(M)} I_{f}, x \mapsto(f(x))_{f \in \mathcal{S}^{*}(M)}$ is a topological embedding.

To prove this it is enough to check (see [Mu, Ch. 4]) that the ring $\mathcal{S}^{*}(M)$ separates points and closed subsets of $M$. Indeed, given a point $p \in M$ and a closed subset $C \subset M$, let $B$ be the intersection of $M$ with an open ball centered at $p$ of small enough radius, such that $B \cap C$ is empty. Then, $K=M \backslash B$ is a closed semialgebraic subset of $M$ containing $C$ and $p \notin K$. From 2.4 applied to the disjoint closed semialgebraic subsets $C_{1}=K$ and $C_{2}=\{p\}$ of $M$, there exists $f \in \mathcal{S}^{*}(M)$ such that $f(p)=1$ and $\left.f\right|_{K} \equiv 0$. Hence, $f$ separates $p$ and $C$, and so $\varphi$ is an embedding. (4.42) Let us define $\beta_{\mathrm{s}}^{\wedge} M$ as the closure of $\varphi(M)$ in $\prod_{f \in \mathcal{S}^{*}(M)} I_{f}$, which is a compact set, and let us see that $\left(\beta_{\mathrm{s}}^{\wedge} M, \varphi\right)$ is a semialgebraically complete compactification of $M$. Indeed, given $g \in \mathcal{S}^{*}(M)$ the projection

$$
\pi_{g}: \beta_{\mathrm{s}}^{\wedge} M \rightarrow I_{g} \subset \mathbb{R},\left(x_{f}\right)_{f \in \mathcal{S}^{*}(M)} \rightarrow x_{g}
$$

is a continuous function and $\pi_{g} \circ \varphi=g$.

4.4.3) The compactification $\left(\beta_{\mathrm{s}}^{\wedge} M, \varphi\right)$ is the smallest among the semialgebraically complete compactifications of $M$ (see 4.3). To prove this, let $(X, \psi)$ be another semialgebraically complete compactification of $M$. For each $f \in \mathcal{S}^{*}(M)$, let us denote $\hat{f}: X \rightarrow \mathbb{R}$ as the unique continuous function such that $\widehat{f} \circ \psi=f$. The continuous map

$$
\Psi: X \rightarrow \prod_{f \in \mathcal{S}^{*}(M)} I_{f}, x \mapsto(\widehat{f}(x))_{f \in \mathcal{S}^{*}(M)}
$$

satisfies $\Psi \circ \psi=\varphi$, and we just need to check that $\operatorname{im} \Psi=\beta_{\mathrm{s}}^{\wedge} M$. This is obvious since $X$ is compact, $\psi(M)$ and $\varphi(M)$ are dense in $X$ and $\beta_{\mathrm{s}}^{\wedge} M$, respectively, and $\Psi \circ \psi=\varphi$.

Because of 4.4114 .413 , we say that $\left(\beta_{\mathrm{s}}^{\wedge} M, \varphi\right)$ is the semialgebraic Stone-Čech compactification of $M$. The adjective semialgebraic refers to the nature of the functions expected to be continuously extended and not to the set $\beta_{\mathrm{s}}^{\wedge} M$, which very rarely is semialgebraic (see 5.17). Clearly, by definition, $\beta_{\mathrm{s}}^{\wedge} M=M$ if and only if $M$ is compact.

(4.5) Semialgebraic compactifications. Let $M \subset \mathbb{R}^{n}$ be a semialgebraic set. A compactification $(X, j)$ of $M$ is said to be a semialgebraic compactification of $M$ if $j$ is a semialgebraic map between the semialgebraic sets $M$ and $X \subset \mathbb{R}^{m}$. Proposition 4.1 provides a large family of semialgebraic compactifications of $M$. 
Our next goal is to compare the semialgebraic Stone-Čech compactification with the semialgebraic ones.

Proposition 4.6. Let $M \subset \mathbb{R}^{n}$ be a semialgebraic set. Then:

(i) Each semialgebraic compactification $(X, j)$ of $M$ satisfies that $(X, j) \preccurlyeq$ $\left(\beta_{\mathrm{s}}^{\wedge} M, \varphi\right)$.

(ii) Let $(Y, i)$ be a compactification of $M$ such that $(X, j) \preccurlyeq(Y, i)$ for every semialgebraic compactification $(X, j)$ of $M$. Then, $\left(\beta_{\mathrm{s}}^{\wedge} M, \varphi\right) \preccurlyeq(Y, i)$.

Proof. (i) Write $j=\left(j_{1}, \ldots, j_{m}\right): M \rightarrow X \subset \mathbb{R}^{m}$. Since $X$ is compact, each component $j_{k} \in \mathcal{S}^{*}(M)$, and we denote by $\widehat{j_{k}}: \beta_{\mathrm{s}}^{\wedge} M \rightarrow \mathbb{R}$ its unique continuous extension, that is, $\hat{j_{k}} \circ \varphi=j_{k}$. Consider the continuous map

$$
\rho=\left(\widehat{j_{1}}, \ldots, \widehat{j_{m}}\right): \beta_{\mathrm{s}}^{\wedge} M \rightarrow \mathbb{R}^{m} .
$$

By definition, $\rho \circ \varphi=j$. Hence, $\rho(\varphi(M))=j(M) \subset X$ and so $\rho\left(\beta_{\mathrm{s}}^{\wedge} M\right) \subset X$. Since $\rho\left(\beta_{\mathrm{s}}^{\wedge} M\right)$ is closed in $X$ and contains the dense subset $j(M)$, we have $\rho\left(\beta_{\mathrm{s}}^{\wedge} M\right)=X$. Therefore, $(X, j) \preccurlyeq\left(\beta_{\mathrm{s}}^{\wedge} M, \varphi\right)$.

(ii) By 4.413, it suffices to prove that $(Y, i)$ is semialgebraically complete. To that end, let $f \in \mathcal{S}^{*}(M)$. By 4.1 there exist a semialgebraic compactification $(X, j)$ of $M$ and a function $F \in \mathcal{S}(X)$ such that $F \circ j=f$. Since $(X, j) \preccurlyeq(Y, i)$ there exists a surjective continuous map $\rho: Y \rightarrow X$ such that $\rho \circ i=j$. Therefore, the function $G=F \circ \rho: Y \rightarrow \mathbb{R}$ satisfies $G \circ i=F \circ \rho \circ i=F \circ j=f$, as wanted.

We showed in 33.1 that $\left(\beta_{\mathrm{s}}^{*} M, \phi\right)$ is a Hausdorff compactification of the semialgebraic set $M$. Let us now see that it is the semialgebraic Stone-Čech compactification of $M$. This way, we get a new model for such a compactification.

Proposition 4.7. Let $M \subset \mathbb{R}^{n}$ be a semialgebraic set and consider the canonical inclusion $\phi: M \hookrightarrow \beta_{\mathrm{s}}^{*} M, p \mapsto \mathfrak{m}_{p}^{*}$. Then, the pair $\left(\beta_{\mathrm{s}}^{*} M, \phi\right)$ is the semialgebraic Stone-Čech compactification of $M$.

Proof. First, observe that for each $f \in \mathcal{S}^{*}(M)$ the map $\widehat{f}: \beta_{\mathrm{s}}^{*} M \rightarrow \mathbb{R}, \mathfrak{m}^{*} \mapsto f+\mathfrak{m}^{*}$ is continuous and $f=\widehat{f} \circ \phi$ (see 3.6). In particular $\left(\beta_{\mathrm{s}}^{*} M, \phi\right)$ is semialgebraically complete. Next, we show that $\beta_{\mathrm{s}}^{*} M \cong \beta_{\mathrm{s}}^{\wedge} M$, via a homeomorphism $\Psi$ such that $\Psi \circ \phi=\varphi$, where $\varphi$ is the topological embedding defined in 4.411. With the notation of 4.4, observe that for each $f \in \mathcal{S}^{*}(M)$,

$$
\widehat{f}\left(\beta_{\mathrm{s}}^{*} M\right)=\widehat{f}\left(\mathrm{Cl}_{\beta_{\mathrm{s}}^{*} M}(\phi(M))\right) \subset \mathrm{Cl}_{\mathbb{R}}(\widehat{f}(\phi(M)))=\mathrm{Cl}_{\mathbb{R}}(f(M)) \subset I_{f} .
$$

Moreover, the family of continuous functions $\left\{\widehat{f}: f \in \mathcal{S}^{*}(M)\right\}$ separates points and closed subsets of $\beta_{\mathrm{s}}^{*} M$. Indeed, let $\mathfrak{n}^{*} \in \beta_{\mathrm{s}}^{*} M$ and let $C \subset \beta_{\mathrm{s}}^{*} M$ be a closed subset of $\beta_{\mathrm{s}}^{*} M$ such that $\mathfrak{n}^{*} \notin C$. Thus, there exists $f \in \mathcal{S}^{*}(M)$ such that $\mathfrak{n}^{*} \in \mathcal{D}_{\beta_{\mathrm{s}}^{*} M}(f) \subset$ $\beta_{\mathrm{s}}^{*} M \backslash C$. Clearly, $f$ satisfies $\left.\widehat{f}\right|_{C} \equiv 0$ and $\widehat{f}\left(\mathfrak{n}^{*}\right) \neq 0$. Therefore, the map

$$
\Psi: \beta_{\mathrm{s}}^{*} M \rightarrow \prod_{f \in \mathcal{S}^{*}(M)} I_{f}, \mathfrak{m}^{*} \mapsto\left(\widehat{f}\left(\mathfrak{m}^{*}\right)\right)_{f \in \mathcal{S}^{*}(M)}
$$

is an embedding.

To check the equality $\Psi \circ \phi=\varphi$, observe that for each point $p \in M$ and each function $f \in \mathcal{S}^{*}(M)$ we know that $\widehat{f}\left(\mathfrak{m}_{p}^{*}\right)=f+\mathfrak{m}_{p}^{*}=f(p)$ or, in other words, $\Psi \circ \phi=\varphi$. 
To finish we must just check that $\operatorname{im} \Psi=\beta_{\mathrm{s}}^{\wedge} M$, which follows at once from the compactness of $\beta_{\mathrm{s}}^{*} M$, the equality $\Psi \circ \phi=\varphi$ and the fact that $\phi(M)$ and $\varphi(M)$ are dense subspaces of $\beta_{\mathrm{s}}^{*} M$ and $\beta_{\mathrm{s}}^{\wedge} M$, respectively.

As a straightforward consequence of 3.5 and 4.7 we have the following.

Corollary 4.8. Let $M \subset \mathbb{R}^{n}$ be a semialgebraic set and consider the canonical inclusion $\phi: M \hookrightarrow \beta_{\mathrm{s}} M, p \mapsto \mathfrak{m}_{p}$. Then, the pair $\left(\beta_{\mathrm{s}} M, \phi\right)$ is the semialgebraic Stone-Čech compactification of $M$.

Thus, we have constructed three different models of the semialgebraic StoneCech compactification of a semialgebraic set $M$. We will choose, according to the involved situation, the suitable model to work with, but we will always denote it by $\beta_{\mathrm{s}}^{*} M$. Only if a particular question requires more details will we use a more specific notation.

For the sake of simplicity, given a compactification $(X, j)$ of $M$ we identify $M$ with its image $j(M)$ and we will write $M \subset X$. Moreover, if $\left(X_{1}, j_{1}\right) \preccurlyeq\left(X_{2}, j_{2}\right)$, there exists a continuous surjective map $\rho: X_{2} \rightarrow X_{1}$ which commutes with the inclusions. After our identification, we will say that $\rho$ is the identity on $M$. Moreover, the remainder $\beta_{\mathrm{s}}^{*} M \backslash M$ of the semialgebraic Stone-Čech compactification of $M$ will be denoted by $\partial M$.

Let us now see an example of a semialgebraic Stone-Čech compactification that will be used to determine the semialgebraic Stone-Čech compactification of semialgebraic curves.

Example 4.9. If $M=[0,1)$ or $(0,1)$, then $\beta_{\mathrm{s}}^{*} M \cong[0,1]$.

Proof. We just consider the case $M=[0,1)$ because the other one is similar. It is clear that $X=[0,1]$ is a semialgebraic compactification of $M$. By [BCR, 2.5.3], each bounded semialgebraic function $f$ defined on $[0,1)$ can be extended to $[0,1]$ just taking $f(1)=\lim _{t \rightarrow 1} f(t)$. Thus, $X$ is semialgebraically complete. Hence, by 4.4. there exists a continuous surjective map $\rho:[0,1] \rightarrow \beta_{\mathrm{s}}^{*} M$. Moreover, by 4.3(i), $\rho(\{1\})=\rho(X \backslash M)=\beta_{\mathrm{s}}^{*} M \backslash M=\partial M$. Hence, $\partial M$ is a singleton, and so $\rho$ is a continuous bijective map from a compact space to a Hausdorff space. Therefore, $\rho$ is a homeomorphism and $\beta_{\mathrm{s}}^{*} M \cong[0,1]$.

The compactification $\beta_{\mathrm{s}}^{*} M$ being Hausdorff, it is a normal topological space, that is, two closed disjoint subsets can be separated by a continuous function. However, since we are dealing with semialgebraic objects it is desirable to separate closed disjoint subsets of $\beta_{\mathrm{s}}^{*} M$ by means of the continuous extension to $\beta_{\mathrm{s}}^{*} M$ of some $f \in \mathcal{S}^{*}(M)$.

Proposition 4.10. Let $M \subset \mathbb{R}^{n}$ be a semialgebraic set. Given two closed disjoint subsets $K_{1}$ and $K_{2}$ of $\beta_{\mathrm{s}}^{*} M$ there exists a bounded semialgebraic function $f: M \rightarrow \mathbb{R}$ such that $\left.\widehat{f}\right|_{K_{1}} \equiv 0$ and $\left.\widehat{f}\right|_{K_{2}} \equiv 1$.

Proof. The open subset $\beta_{\mathrm{s}}^{*} M \backslash K_{1}$ is a union $\bigcup_{i \in I} \mathcal{D}_{\beta_{\mathrm{s}}^{*} M}\left(g_{i}\right)$ with $g_{i} \in \mathcal{S}^{*}(M)$. Moreover, since $\beta_{\mathrm{s}}^{*} M$ is compact, $K_{2} \subset \beta_{\mathrm{s}}^{*} M \backslash K_{1}$ is compact too, and so there exists a finite subset $J$ of $I$ with $K_{2} \subset \bigcup_{i \in J} \mathcal{D}_{\beta_{\mathrm{s}}^{*} M}\left(g_{i}\right)=\mathcal{D}_{\beta_{\mathrm{s}}^{*} M}(g)$, where $g=\sum_{i \in J} g_{i}^{2}$. Therefore,

$$
r=\min \left\{\widehat{g}(\mathfrak{m}): \mathfrak{m} \in K_{2}\right\}>0 \quad \text { and } \quad \mathcal{D}_{\beta_{\mathrm{s}}^{*} M}(g) \subset \bigcup_{i \in I} \mathcal{D}_{\beta_{\mathrm{s}}^{*} M}\left(g_{i}\right)=\beta_{\mathrm{s}}^{*} M \backslash K_{1} .
$$


Thus, the bounded semialgebraic function $f: M \rightarrow \mathbb{R}, x \mapsto \frac{\min \{r, g(x)\}}{r}$ satisfies $\left.\widehat{f}\right|_{K_{1}} \equiv 0$ and $\left.\widehat{f}\right|_{K_{2}} \equiv 1$.

(4.11) Locally compact semialgebraic sets. In general topology the locally compact Hausdorff spaces are characterized as those spaces which admit a Hausdorff compactification by a single point ([Mu, 3.29.1]). For our purposes, it will be profitable to use semialgebraic compactifications by a single point of locally compact semialgebraic sets. On the other hand, it must be pointed out that the local closedness has been revealed, in the semialgebraic setting, as an important property for the validity of results which are in the core of semialgebraic geometry. Recall that the locally closed subsets of a locally compact topological space coincide with the locally compact ones (see for instance [Bo, §9.7, Prop. 1 2-13]). Namely,

Lemma 4.12. Let $T$ be a Hausdorff and locally compact topological space. Given $M \subset T$, the following conditions are equivalent:

(i) $M$ is locally closed.

(ii) $M=U \cap \mathrm{Cl}_{T}(M)$, where $U=T \backslash\left(\mathrm{Cl}_{T}(M) \backslash M\right)$ is an open subset of $T$.

(iii) $M$ is a locally compact space.

Remark 4.13. If $M \subset \mathbb{R}^{n}$ is a semialgebraic set, then the sets $\mathrm{Cl}_{\mathbb{R}^{n}}(M)$ and $U=\mathbb{R}^{n} \backslash$ $\left(\mathrm{Cl}_{\mathbb{R}^{n}}(M) \backslash M\right)$ are also semialgebraic. Thus, if $M$ is a locally compact semialgebraic set, it can be written as the intersection of a closed and an open semialgebraic subsets of $\mathbb{R}^{n}$.

Corollary 4.14. Let $M \subset \mathbb{R}^{n}$ be a semialgebraic set. The following statements are equivalent:

(i) $M$ is locally compact.

(ii) The remainder $X \backslash j(M)$ of each Hausdorff compactification $(X, j)$ of $M$ is a closed subset of $X$.

(iii) There exists a Hausdorff compactification $(X, j)$ of $M$ whose remainder $X \backslash j(M)$ is a closed subset of $X$.

Next, we recall some properties of the largest locally compact and dense subset $M_{\mathrm{lc}}$ of a semialgebraic set $M$ whose construction is a main goal of [Fe1, 3.8].

Theorem 4.15. Let $M \subset \mathbb{R}^{n}$ be a semialgebraic set. Define

$$
\rho_{0}(M)=\mathrm{Cl}_{\mathbb{R}^{n}}(M) \backslash M \quad \text { and } \quad \rho_{1}(M)=\rho_{0}\left(\rho_{0}(M)\right)=\mathrm{Cl}_{\mathbb{R}^{n}}\left(\rho_{0}(M)\right) \cap M .
$$

Then, the semialgebraic set $M_{\mathrm{lc}}=M \backslash \rho_{1}(M)=\mathrm{Cl}_{\mathbb{R}^{n}}(M) \backslash \mathrm{Cl}_{\mathbb{R}^{n}}\left(\rho_{0}(M)\right)$ is the largest locally compact and dense subset of $M$ and coincides with the set of points of $M$ which have a compact neighbourhood in $M$.

Corollary 4.16. Let $M \subset \mathbb{R}^{n}$ be a bounded noncompact semialgebraic set. Then, the semialgebraic set $\mathrm{Cl}_{\mathbb{R}^{n}}(M) \backslash\left(\mathrm{Cl}_{\mathbb{R}^{n}}\left(\rho_{1}(M)\right) \cup M\right)$ is nonempty.

Proof. Suppose by way of contradiction that $\mathrm{Cl}_{\mathbb{R}^{n}}(M)=\mathrm{Cl}_{\mathbb{R}^{n}}\left(\rho_{1}(M)\right) \cup M$. Subtracting $M$ on both sides we get

$$
\rho_{0}(M)=\mathrm{Cl}_{\mathbb{R}^{n}}(M) \backslash M=\mathrm{Cl}_{\mathbb{R}^{n}}\left(\rho_{1}(M)\right) \backslash M \subset \mathrm{Cl}_{\mathbb{R}^{n}}\left(\rho_{1}(M)\right)=\mathrm{Cl}_{\mathbb{R}^{n}}\left(\rho_{0}\left(\rho_{0}(M)\right)\right) .
$$

But this is impossible, because by [BCR, 2.8.13],

$$
\operatorname{dim} \mathrm{Cl}_{\mathbb{R}^{n}}\left(\rho_{0}\left(\rho_{0}(M)\right)\right)=\operatorname{dim} \rho_{0}\left(\rho_{0}(M)\right)<\operatorname{dim} \rho_{0}(M),
$$

and we are done. 
Now, we show that each locally compact semialgebraic set admits a semialgebraic compactification by a single point. Namely,

Lemma 4.17 (Semialgebraic compactification by a single point). Let $M \subset \mathbb{R}^{n}$ be a locally compact but not compact semialgebraic set. Then,

(i) There exists a semialgebraic homeomorphism between $M$ and a closed semialgebraic subset of $\mathbb{R}^{n+1}$.

(ii) There exists a semialgebraic homeomorphism $\varphi: M \rightarrow \varphi(M) \subset \mathbb{R}^{n+2}$ such that $\varphi(M) \subset \mathbb{R}^{n+2} \backslash\{0\}$ and $\mathrm{Cl}_{\mathbb{R}^{n+2}}(\varphi(M))=\varphi(M) \cup\{0\}$ is a compact set.

Proof. (i) Since locally compact semialgebraic subsets of $\mathbb{R}^{n}$ are locally closed, the statement follows from BCR, 2.2.9].

(ii) Identify $\mathbb{R}^{n+1}$ with the hyperplane $\left\{x_{n+2}=0\right\} \subsetneq \mathbb{R}^{n+2}$ and consider a stereographic projection

$$
\phi: S=\left\{x \in \mathbb{R}^{n+2}: \sum_{i=1}^{n+1} x_{i}^{2}+\left(x_{n+2}+1\right)^{2}=1\right\} \backslash\{0\} \rightarrow \mathbb{R}^{n+1} .
$$

Let us denote $\psi: M \rightarrow \psi(M) \subset \mathbb{R}^{n+1}$ as a semialgebraic homeomorphism, where $\psi(M)$ is a closed semialgebraic subset of $\mathbb{R}^{n+1}$. Hence, the composition $\varphi=\phi^{-1} \circ \psi$ does the job.

Finally in this section we present a class of semialgebraic sets whose semialgebraic Stone-Cech compactification is (homeomorphic to) a semialgebraic set. In fact, as we will prove in 5.17, this is the class of those semialgebraic sets whose semialgebraic Stone-Cech compactification is a semialgebraic set.

(4.18) For the time being, given a semialgebraic set $M \subset \mathbb{R}^{n}$, we denote by $M^{\geq 2}=\left\{p \in M: \operatorname{dim}_{p} M \geq 2\right\}$ the set of points of $M$ of local dimension $\geq 2$. We refer the reader to $\mathrm{BCR}, 2.8 .10-11]$ for further details about the local dimension of semialgebraic sets. This set $M^{\geq 2}$ turns out to be semialgebraic, and there exists a closed semialgebraic subset $L \subset M$ of dimension $\leq 1$ such that $M=M^{\geq 2} \cup L$ and $M^{\geq 2} \cap L$ is either empty or a finite set (see for instance [FG4, 2.7(ii)]). In fact, $L$ is the closure in $M$ of the set of points of $M$ of dimension $\leq 1$. Also recall that by [FG4, 2.7(iii)], if $M \geq 2$ is compact, then $M$ is locally compact.

Proposition 4.19. Let $M \subset \mathbb{R}^{n}$ be a semialgebraic set such that $M \geq 2$ is compact. Then, $\beta_{\mathrm{s}}^{*} M$ is homeomorphic to a semialgebraic set and $\partial M$ is a finite set.

Proof. If $M$ is compact there is nothing to prove. Thus, in what follows we assume that $M$ is not compact. By 4.18, we may write $M=M^{\geq 2} \cup L$, where the semialgebraic set $L$ is the closure in $M$ of the set of points in $M$ of local dimension $\leq 1$; in particular, $M^{\geq 2} \cap L$ is either empty or a finite set. Since $M^{\geq 2}$ is compact, $M$ is locally compact and we may assume, by 4.17 that $M \cup\{0\}$ is compact. Next, by [BCR, 9.3.6], there exist $\varepsilon>0$ and a semialgebraic homeomorphism $\varphi: \overline{\mathbb{B}}_{n}(0, \varepsilon) \rightarrow \overline{\mathbb{B}}_{n}(0, \varepsilon)$ such that:

(i) $\|\varphi(y)\|=\|y\|$ for every $y \in \overline{\mathbb{B}}_{n}(0, \varepsilon)$,

(ii) $\left.\varphi\right|_{\mathbb{S}^{n-1}(0, \varepsilon)}$ is the identity map,

(iii) $\varphi^{-1}\left(M \cap \overline{\mathbb{B}}_{n}(0, \varepsilon)\right)$ is the cone with vertex 0 and basis $M \cap \mathbb{S}^{n-1}(0, \varepsilon)$, with its vertex excluded. 
Let us also denote $\varphi: \mathbb{R}^{n} \rightarrow \mathbb{R}^{n}$ as the extension of such homeomorphism to the whole $\mathbb{R}^{n}$ using the identity map outside $\overline{\mathbb{B}}_{n}(0, \varepsilon)$, and identify $M$ with $\varphi(M)$. Notice that since $M^{\geq 2}$ is compact, $\{0\}$ is adherent to $L$ but not to $M^{\geq 2}$, and so $M \cap \mathbb{S}^{n-1}(0, \varepsilon)$ is a finite set. This allows us to write $M$ as the union of a compact semialgebraic set $Z=M \cap\left(\mathbb{R}^{n} \backslash \mathbb{B}_{n}(0, \varepsilon)\right)$ and finitely many segments $C_{1}, \ldots, C_{r}$, which are Nash diffeomorphic to $[0,1)$ and whose closure connects the irreducible components of the finite semialgebraic set $Z \cap \mathbb{S}^{n-1}(0, \varepsilon)$ with the origin. Now consider the semialgebraic set $C$ written as the disjoint union $C=C_{1} \sqcup \cdots \sqcup C_{r}$ of its connected components $C_{i}$. Since $Z$ is compact, $C$ is closed in $M$ and $M=Z \cup C$, we have

$$
\beta_{\mathrm{s}}^{*} M=\mathrm{Cl}_{\beta_{\mathrm{s}}^{*} M}(M)=\mathrm{Cl}_{\beta_{\mathrm{s}}^{*} M}(C) \cup \mathrm{Cl}_{\beta_{\mathrm{s}}^{*} M}(Z)=\mathrm{Cl}_{\beta_{\mathrm{s}}^{*} M}(C) \cup Z
$$

and $\mathrm{Cl}_{\beta_{\mathrm{s}}^{*} M}(C) \cong \beta_{\mathrm{s}}^{*} C$ (see 3.144). To simplify notation we identify the last two spaces and write $\beta_{\mathrm{s}}^{*} M=\beta_{\mathrm{s}}^{*} C \cup Z$. Thus, since $C$ is closed in $M$, we have

$$
\partial M=\beta_{\mathrm{s}}^{*} M \backslash M=\left(\beta_{\mathrm{s}}^{*} C \cup Z\right) \backslash M=\beta_{\mathrm{s}}^{*} C \backslash C .
$$

On the other hand, by 3.115, $\beta_{\mathrm{s}}^{*} C$ can be written as the disjoint union

$$
\beta_{\mathrm{s}}^{*} C=\beta_{\mathrm{s}}^{*} C_{1} \sqcup \cdots \sqcup \beta_{\mathrm{s}}^{*} C_{r},
$$

and, by 4.9, each $\beta_{\mathrm{s}}^{*} C_{i}$ is homeomorphic to $[0,1]$ and $\partial C_{i}$ is a singleton. Moreover, since each $C_{i}$ is closed in $C$,

$$
\partial M=\partial C=\beta_{\mathrm{s}}^{*} C \backslash C=\bigsqcup_{i=1}^{r} \beta_{\mathrm{s}}^{*} C_{i} \backslash C_{i}=\bigsqcup_{i=1}^{r} \partial C_{i},
$$

and, in particular, $\partial M$ is a finite set. Even more, $M$ is semialgebraically homeomorphic to $M_{0}=M \backslash \mathbb{B}_{n}(0, \varepsilon / 2)$ and $\beta_{\mathrm{s}}^{*} M_{0} \cong \beta_{\mathrm{s}}^{*} M$ is homeomorphic to $\operatorname{Cl}_{\mathbb{R}^{n+2}}\left(M_{0}\right)$, which is a semialgebraic set.

Remark 4.20. Roughly speaking, if the semialgebraic subset $M^{\geq 2} \subset M$ is compact, then the semialgebraic Stone-Cech compactification $\beta_{\mathrm{s}}^{*} M$ is obtained by adding to $M$ a different ending point at each open half-branch of $M$. In particular, if $M$ is a semialgebraic curve, then $M^{\geq 2}=\varnothing$ is a compact set. Thus, 4.19 applies to $M$ and so $\beta_{\mathrm{s}}^{*} M$ is homeomorphic to a semialgebraic set.

\section{Topological properties of the Remainder}

The analysis of the topological properties of the remainder of the Stone-Cech compactification of a completely regular topological space comes back to the pioneer work of Gillman-Jerison (see [GJ, $\S 6, \S 9, \S 10, \S 14]$ ). We study in this last section the same kind of properties in the semialgebraic context, paying special attention to the finiteness of the number of connected components of $\partial M=\beta_{\mathrm{s}}^{*} M \backslash M$. Notice that the remainder is rarely a semialgebraic set. In fact, this happens if and only if $\partial M$ is a finite set (see 5.17).

(5.1) Connected components of $\partial M$. We first prove the finiteness of the number of connected components of the remainder $\partial M$ of the semialgebraic StoneČech compactification of a semialgebraic set $M$. In fact, we prove a stronger result, namely, the existence of a semialgebraic compactification $X$ of $M$ whose remainder $X \backslash M$ shares the number of connected components with $\partial M$. Moreover, in case $M$ is locally compact we show that such a number coincides with the number of 
connected components of the germ at infinity of $M$, where it is seen as a subset of its one point semialgebraic compactification (see 4.17).

We will first observe that in fact the number of connected components of $\partial M$ is greater than or equal to the number of connected components of $X \backslash M$ for every semialgebraic compactification $X$ of $M$.

Lemma 5.2. Let $X \subset \mathbb{R}^{m}$ be a semialgebraic compactification of a semialgebraic set $M \subset \mathbb{R}^{n}$. Then, the number of connected components of $\partial M$ is greater than or equal to the number of connected components of $X \backslash M$.

Proof. By 4.6, there exists a surjective continuous map $\rho: \beta_{\mathrm{s}}^{*} M \rightarrow X$ which is the identity over $M$. By 4.3, the restriction $\left.\rho\right|_{\partial M}: \partial M \rightarrow X \backslash M$ is continuous and surjective. Therefore, the inverse image of each connected component of $X \backslash M$ is open and closed in $\partial M$; hence, it is a union of connected components of $\partial M$. This proves the result.

For our purposes it will be fruitful to use some properties concerning the density in $\partial M$ of the collection $\widetilde{\partial} M$ of all free maximal ideals associated to semialgebraic paths. Namely

(5.3) Free maximal ideals associated to semialgebraic paths. Let $M \subset \mathbb{R}^{n}$ be a semialgebraic set and let $\alpha:(0,1] \rightarrow M$ be a semialgebraic path which cannot be extended to a continuous map $[0,1] \rightarrow M$. By [FG1, 2.5], the set

$$
\mathfrak{m}_{\alpha}^{*}=\left\{f \in \mathcal{S}^{*}(M): \lim _{t \rightarrow 0}(f \circ \alpha)(t)=0\right\}
$$

is a maximal ideal of $\mathcal{S}^{*}(M)$. Since $\alpha$ cannot be extended to a continuous path $[0,1] \rightarrow M$, the ideal $\mathfrak{m}_{\alpha}^{*}$ is free.

The collection of all free maximal ideals of $\mathcal{S}^{*}(M)$ defined as above is denoted by $\widetilde{\partial} M$ (see $\mathrm{Fe} 2$ for further details), and as we will see it is a dense subset of $\partial M$. In fact, we have:

Proposition 5.4. Let $M \subset \mathbb{R}^{n}$ be a semialgebraic set. Then:

(i) Let $f_{1}, \ldots, f_{r} \in \mathcal{S}^{*}(M)$, and for each $i=1, \ldots, r$ let $\widehat{f}_{i}: \beta_{\mathrm{s}}^{*} M \rightarrow \mathbb{R}$ be the unique continuous extension of $f_{i}$ to $\beta_{\mathrm{s}}^{*} M$. Then, $\left(\widehat{f}_{1}, \ldots, \widehat{f}_{r}\right)(\widetilde{\partial} M)=$ $\left(\widehat{f}_{1}, \ldots, \widehat{f}_{r}\right)(\partial M)$.

(ii) The set $\widetilde{\partial} M$ is dense in $\partial M$.

Proof. All along the proof we may assume, by 2.1, that $M \subset \mathbb{B}_{n}(0,1)$.

(i) Let $\mathfrak{m}^{*} \in \partial M$. Since $\mathrm{Cl}_{\mathbb{R}^{n}}(M)$ is a semialgebraic compactification of $M$, there exists a surjective continuous map $\rho: \beta_{\mathrm{s}}^{*} M \rightarrow \mathrm{Cl}_{\mathbb{R}^{n}}(M)$ which is the identity on $M$. By 4.3. $p_{0}=\rho\left(\mathfrak{m}^{*}\right) \in \mathrm{Cl}_{\mathbb{R}^{n}}(M) \backslash M$. Consider the proper map

$$
\Psi=(\rho, \widehat{f}): \beta_{\mathrm{s}}^{*} M \rightarrow \mathbb{R}^{n+r}
$$

where we abbreviate $f=\left(f_{1}, \ldots, f_{r}\right)$ and $\widehat{f}=\left(\widehat{f}_{1}, \ldots, \widehat{f}_{r}\right)$. The image of the restriction $\left.\Psi\right|_{M}$ is the graph $\Gamma$ of $f$ because $\widehat{f}_{i}\left(\mathfrak{m}_{p}^{*}\right)=f_{i}(p)$ for each $p \in M$, and since $\Psi$ is proper one gets

$$
\operatorname{im} \Psi=\Psi\left(\mathrm{Cl}_{\beta_{\mathrm{s}}^{*} M}(M)\right)=\mathrm{Cl}_{\mathbb{R}^{n+r}}(\Psi(M))=\mathrm{Cl}_{\mathbb{R}^{n+r}}(\Gamma) .
$$

Let $q_{0}=\Psi\left(\mathfrak{m}^{*}\right)=\left(\rho\left(\mathfrak{m}^{*}\right), \widehat{f}\left(\mathfrak{m}^{*}\right)\right)=\left(p_{0}, a_{0}\right) \in \mathrm{Cl}_{\mathbb{R}^{n+r}}(\Gamma) \backslash \Gamma \subset \mathbb{R}^{n} \times \mathbb{R}^{r}$; see 4.3(i). By the Curve Selection Lemma BCR, 2.5.5], there exist semialgebraic paths 
$\alpha:[0,1] \rightarrow \mathbb{R}^{n}$ and $\nu:[0,1] \rightarrow \mathbb{R}^{r}$ such that $\alpha((0,1]) \subset M,\left.\nu\right|_{(0,1]}=\left.(f \circ \alpha)\right|_{(0,1]}$ and $(\alpha(0), \nu(0))=\left(p_{0}, a_{0}\right)=q_{0}$. Finally, we deduce that

$$
a_{0}=\nu(0)=\lim _{t \rightarrow 0} \nu(t)=\lim _{t \rightarrow 0}(f \circ \alpha)(t)=\widehat{f}\left(\mathfrak{m}_{\alpha}^{*}\right),
$$

where $\mathfrak{m}_{\alpha}^{*} \in \widetilde{\partial} M$ because $\lim _{t \rightarrow 0} \alpha(t)=p_{0} \notin M$.

(ii) We have to check that for every $f \in \mathcal{S}^{*}(M)$ such that $\mathcal{D}_{\beta_{\mathrm{s}}^{*} M}(f) \not \subset M$, the intersection $\mathcal{D}_{\beta_{\mathrm{s}}^{*} M}(f) \cap \widetilde{\partial} M \neq \varnothing$. Otherwise, $\widetilde{\partial} M \subset \mathcal{Z}_{\beta_{\mathrm{s}}^{*} M}(f)$, and we consider the continuous function $\widehat{f}: \beta_{\mathrm{s}}^{*} M \rightarrow \mathbb{R}$ such that $\left.\widehat{f}\right|_{M}=f$. Then, by part (i), $\{0\}=\widehat{f}(\widetilde{\partial} M)=\widehat{f}(\partial M)$, or equivalently, $\mathcal{D}_{\beta_{\mathrm{s}}^{*} M}(f) \subset M$, a contradiction.

Now, we compute the number of connected components of $\partial M$ for a semialgebraic set $M \subset \mathbb{R}^{n}$ which is comprised in between an open ball and its closure in $\mathbb{R}^{n}$. Namely, we obtain the following result whose proof is inspired in [GJ, 6.10].

Lemma 5.5. Let us abbreviate $B=\mathbb{B}_{n}(0,1)$ and $\bar{B}=\overline{\mathbb{B}}_{n}(0,1)$, and let $M \subset \mathbb{R}^{n}$ be a semialgebraic set such that $B \subset M \subset \bar{B}$. Then, the number of connected components of $\partial M$ equals the number of connected components of the semialgebraic set $\bar{B} \backslash M$.

Proof. First, by 5.2. the number of connected components of $\bar{B} \backslash M$ is less than or equal to the number of connected components of $\partial M$.

To prove the converse inequality let $\rho: \beta_{\mathrm{s}}^{*} M \rightarrow \bar{B}$ be the unique continuous surjective map whose restriction to $M$ is the identity. It is enough to check that given two points $\mathfrak{m}_{1}^{*}, \mathfrak{m}_{2}^{*} \in \partial M$ such that $p=\rho\left(\mathfrak{m}_{1}^{*}\right)$ and $q=\rho\left(\mathfrak{m}_{2}^{*}\right)$ belong to the same connected component $C$ of $\bar{B} \backslash M$, then $\mathfrak{m}_{1}^{*}$ and $\mathfrak{m}_{2}^{*}$ belong to the same connected component of $\partial M$.

Indeed, by [BCR, 2.4.5, 2.5.13], there exists a semialgebraic path $\alpha:[0,1] \rightarrow C$ connecting $p$ with $q$. Let $Y=\alpha([0,1]) \subset C$, which is a compact semialgebraic set. By 2.2, there exists $u \in \mathcal{S}^{*}\left(\mathbb{R}^{n}\right)$ such that $Z_{\mathbb{R}^{n}}(u)=Y$. It suffices to prove that the set $T=\mathcal{Z}_{\beta_{\mathrm{s}}^{*} M}\left(\left.u\right|_{M}\right)$ is connected, since it contains $\mathfrak{m}_{1}^{*}$ and $\mathfrak{m}_{2}^{*}$. This last is so because

$$
\mathfrak{m}_{i}^{*} \in \rho^{-1}\left(Z_{\mathbb{R}^{n}}(u)\right)=\mathcal{Z}_{\beta_{\mathrm{s}}^{*} M}(u \circ \rho)=\mathcal{Z}_{\beta_{\mathrm{s}}^{*} M}\left(\left.u\right|_{M}\right)=T .
$$

Note that $T \subset \partial M$, because $Z_{M}(u)=\varnothing$. Moreover, $T$ is compact because it is a closed subset of $\beta_{\mathrm{s}}^{*} M$.

(5.51) Suppose that $T$ is not connected. Then, there exist two disjoint nonempty closed subsets $C_{1}, C_{2} \subsetneq T$ such that $T=C_{1} \sqcup C_{2}$. Of course, both $C_{1}$ and $C_{2}$ are also open subsets in $T$. Thus, there exists a family of semialgebraic functions $\left\{f_{i}\right\}_{i \in I} \subset \mathcal{S}^{*}(M)$ such that $C_{1}=T \cap \bigcup_{i \in I} \mathcal{D}_{\beta_{\mathrm{s}}^{*} M}\left(f_{i}\right)$. Since $C_{1}$ is compact, there exists a finite set $J \subset I$ such that

$$
C_{1}=T \cap \bigcup_{i \in J} \mathcal{D}_{\beta_{\mathrm{s}}^{*} M}\left(f_{i}\right)=T \cap \mathcal{D}_{\beta_{\mathrm{s}}^{*} M}(f),
$$

where $f=\sum_{i \in J} f_{i}^{2} \in \mathcal{S}^{*}(M)$. Therefore, the semialgebraic function $g_{2}=u^{2}+f^{2} \in$ $\mathcal{S}^{*}(M)$ satisfies $C_{2}=\mathcal{Z}_{\beta_{\mathrm{s}}^{*} M}\left(g_{2}\right)$. With the same argument, there exists a function $g_{1} \in \mathcal{S}^{*}(M)$ such that $C_{1}=\mathcal{Z}_{\beta_{\mathrm{s}}^{*} M}\left(g_{1}\right)$. Since $C_{1}$ and $C_{2}$ are disjoint, the sum $g_{1}^{2}+g_{2}^{2}$ is a unit in $\mathcal{S}^{*}(M)$. Therefore, its inverse $h=1 /\left(g_{1}^{2}+g_{2}^{2}\right) \in \mathcal{S}^{*}(M)$. The unique continuous extension $\widehat{h_{1}}: \beta_{\mathrm{s}}^{*} M \rightarrow \mathbb{R}$ of the bounded semialgebraic function $h_{1}=g_{1}^{2} h=g_{1}^{2} /\left(g_{1}^{2}+g_{2}^{2}\right) \in \mathcal{S}^{*}(M)$ vanishes identically on $C_{1}$ and $\left.\widehat{h_{1}}\right|_{C_{2}} \equiv 1$. By 5.4. 
there exist two semialgebraic paths $\alpha_{1}, \alpha_{2}:[0,1] \rightarrow \mathbb{R}^{n}$ such that $\alpha_{i}((0,1]) \subset M$, $\alpha_{1}(0)=p, \alpha_{2}(0)=q$ and

$$
\lim _{s \rightarrow 0}\left(h_{1} \circ \alpha_{i}\right)(s)= \begin{cases}0 & \text { if } i=1, \\ 1 & \text { if } i=2 .\end{cases}
$$

(5.5) 2) For each positive integer $m \geq 2$, consider the open semialgebraic neighbourhood $V_{m}=\{x \in M$ : $\operatorname{dist}(x, Y)<1 / m\}$ of $Y$ in $M$. Pick two points $a_{m} \in$ $\mathbb{B}_{n}(p, 1 / m) \cap \alpha_{1}((0,1])$ and $b_{m} \in \mathbb{B}_{n}(q, 1 / m) \cap \alpha_{2}((0,1])$ satisfying

$$
\alpha_{1}\left(\left(0, \alpha_{1}^{-1}\left(a_{m}\right)\right]\right) \subset \mathbb{B}_{n}(p, 1 / m) \quad \text { and } \quad \alpha_{2}\left(\left(0, \alpha_{2}^{-1}\left(b_{m}\right)\right]\right) \subset \mathbb{B}_{n}(q, 1 / m) .
$$

Consider, for each $\lambda \in[0,1]$, the connected semialgebraic set $Y_{\lambda}=\{\lambda x: x \in Y\}$, which is semialgebraically path connected by BCR 2.5.13]. Take $\lambda_{m}=1-\frac{1}{2 m}$ and choose two points $c_{m} \in \mathbb{B}_{n}(p, 1 / m) \cap Y_{\lambda_{m}}$ and $d_{m} \in \mathbb{B}_{n}(q, 1 / m) \cap Y_{\lambda_{m}}$. Since both sets $\mathbb{B}_{n}(p, 1 / m) \cap B$ and $\mathbb{B}_{n}(q, 1 / m) \cap B$ are semialgebraically path connected, there exist semialgebraic paths

$$
\eta_{1}:[0,1] \rightarrow \mathbb{B}_{n}(p, 1 / m) \cap B \quad \text { and } \quad \eta_{2}:[0,1] \rightarrow \mathbb{B}_{n}(q, 1 / m) \cap B
$$

which connect, respectively, $a_{m}$ with $c_{m}$ and $b_{m}$ with $d_{m}$. Next, since $Y_{\lambda_{m}}$ is semialgebraically path connected, we find a semialgebraic path $\eta_{3}:[0,1] \rightarrow B$ connecting $c_{m}$ and $d_{m}$. By suitably gluing the previous semialgebraic paths $\left.\alpha_{1}\right|_{\left(0, \alpha_{1}^{-1}\left(a_{m}\right)\right]}$, $\eta_{1}, \eta_{3}, \eta_{2}$ and $\left.\alpha_{2}\right|_{\left(0, \alpha_{2}^{-1}\left(b_{m}\right)\right]}$ we construct, for each $m \geq 2$, a semialgebraic path $\gamma_{m}:(0,1) \rightarrow M$ such that

$$
\operatorname{im} \gamma_{m} \subset V_{m}, \quad \lim _{s \rightarrow 0}\left(h_{1} \circ \gamma_{m}\right)(s)=0, \quad \text { and } \quad \lim _{s \rightarrow 1}\left(h_{1} \circ \gamma_{m}\right)(s)=1 .
$$

Hence, for each $m \geq 2$, there exists a point $q_{m} \in V_{m}$ such that $h_{1}\left(q_{m}\right)=\frac{1}{2}$. By the compactness of $\bar{B}$ the set $Q=\left\{q_{m}\right\}_{m \geq 2}$ has a cluster point $q_{0} \in \bar{B}$. In fact, $q_{0} \in Y$, because $\lim _{m \rightarrow+\infty} d\left(q_{m}, Y\right)=0$ and, of course, we may assume that $\left\{q_{m}\right\}_{m \geq 2}$ converges to $q_{0}$. Thus, $u\left(q_{0}\right)=0$ since $q_{0} \in Y$, and so the sequence $\left\{u\left(q_{m}\right)\right\}_{m \geq 2}$ converges to 0 .

On the other hand, $\beta_{\mathrm{s}}^{*} M$ being compact, there exists a cluster point $\mathfrak{m}^{*} \in \beta_{\mathrm{s}}^{*} M$ of $Q$. Since $\left\{u\left(q_{m}\right)\right\}_{m \geq 2}$ converges to 0 , the unique continuous extension $\widehat{u}: \beta_{\mathrm{s}}^{*} M \rightarrow \mathbb{R}$ of $u$ satisfies $\widehat{u}\left(\mathfrak{m}^{*}\right)=0$. Therefore, $\mathfrak{m}^{*} \in T$, and $\widehat{h_{1}}\left(\mathfrak{m}^{*}\right)=1 / 2$ because $h_{1}\left(q_{m}\right)=$ $1 / 2$ for all $m \geq 2$. This is a contradiction because $\left.h_{1}\right|_{T}$ just takes the values 0 and 1. Thus, $T$ is connected, and we are done.

For our purpose of computing the number of connected components of the remainder $\partial M$ we use the results about triangulations of compact semialgebraic sets already introduced in 2.5

Lemma 5.6. Let $M \subset \mathbb{R}^{n}$ be a semialgebraic set. Then, $\partial M$ has finitely many connected components.

Proof. First, by 2.1 we may assume that $M$ is bounded and so $X=\mathrm{Cl}_{\mathbb{R}^{n}}(M)$ is compact. By 2.6. there exist a finite simplicial complex $K=\left(\sigma_{i}\right)_{i=1}^{p}$ and a semialgebraic homeomorphism $\Phi:|K| \rightarrow X$ such that $M$ is the union of some $\Phi\left(\sigma_{i}^{0}\right)$. We may assume that $\Phi\left(\sigma_{i}^{0}\right) \cap M \neq \varnothing$ exactly for $i=1, \ldots, s$. This implies, by the choice of $|K|$, that $\Phi\left(\sigma_{i}^{0}\right) \subset M$ for $i=1, \ldots, s$. Hence,

$$
\Phi\left(\sigma_{i}^{0}\right) \subset S_{i}=M \cap \Phi\left(\sigma_{i}\right) \subset \Phi\left(\sigma_{i}\right)
$$


for $i=1, \ldots, s$. Note that $S_{i}$ is a closed subset of $M$ for $i=1, \ldots, s$ and that $M=\bigcup_{i=1}^{s} S_{i}$. Then, $\beta_{\mathrm{s}}^{*} M=\bigcup_{i=1}^{s} \mathrm{Cl}_{\beta_{\mathrm{s}}^{*} M}\left(S_{i}\right)$ and since $S_{i}$ is closed in $M$, we get $\partial M=\bigcup_{i=1}^{s}\left(\mathrm{Cl}_{\beta_{\mathrm{s}}^{*} M}\left(S_{i}\right) \backslash S_{i}\right)$. By 3.114 $\mathrm{Cl}_{\beta_{\mathrm{s}}^{*} M}\left(S_{i}\right) \backslash S_{i}$ is homeomorphic to $\beta_{\mathrm{s}}^{*} S_{i} \backslash S_{i}$. Thus, all reduces to check that $\beta_{\mathrm{s}}^{*} S_{i} \backslash S_{i}$ has finitely many connected components for $i=1, \ldots, s$. But this follows from 5.5 and the fact that a simplex is a compact semialgebraic manifold with boundary, semialgebraically homeomorphic to a closed ball of its same dimension.

After 5.2 and 5.6 it is a natural question to decide if the number of connected components of $\partial M$ equals the number of connected components of the remainder of some semialgebraic compactification of $M$. We answer this question in the affirmative in 5.8 whose proof is rather involved. Before that we need a preliminary result.

Lemma 5.7. Let $N \subset M \subset \mathbb{R}^{n}$ be semialgebraic sets such that $N$ is closed and nowhere dense in $M$. If $\partial M \neq \varnothing$, then $\partial M \not \subset \mathrm{Cl}_{\beta_{\mathrm{s}}^{*} M}(N)$.

Proof. First, by 2.1. we may assume that $M$ is bounded. Since $\partial M \neq \varnothing, M$ is not compact, and so there exists a point $p \in \mathrm{Cl}_{\mathbb{R}^{n}}(M) \backslash M$. Moreover, since $M \backslash N$ is dense in $M$, we have $\mathrm{Cl}_{\mathbb{R}^{n}}(M \backslash N)=\mathrm{Cl}_{\mathbb{R}^{n}}(M)$. Thus, by the Curve Selection Lemma $[\mathrm{BCR}, 2.5 .5]$ there exists a semialgebraic path $\alpha:[0,1] \rightarrow \mathbb{R}^{n}$ such that $\alpha(0)=p$ and $C=\alpha((0,1]) \subset M \backslash N$. Note that $C$ is a closed semialgebraic subset of $M$ disjoint with $N$. Hence, by 2.4, there exists $f \in \mathcal{S}^{*}(M)$ such that $\left.f\right|_{C}=0$ and $\left.f\right|_{N}=1$. In particular, the continuous extension $\widehat{f}: \beta_{\mathrm{s}}^{*} M \rightarrow \mathbb{R}$ of $f$ satisfies that $\mathfrak{m}_{\alpha}^{*} \in \mathrm{Cl}_{\beta_{\mathrm{s}}^{*} M}(C) \subset \mathcal{Z}_{\beta_{\mathrm{s}}^{*} M}(\widehat{f})$ and $\mathrm{Cl}_{\beta_{\mathrm{s}}^{*} M}(N) \subset \mathcal{Z}_{\beta_{\mathrm{s}}^{*} M}(\widehat{f}-1)$. Therefore, $\mathfrak{m}_{\alpha}^{*} \in \partial M \backslash \mathrm{Cl}_{\beta_{\mathrm{s}}^{*} M}(N)$, as wanted.

Theorem 5.8 (Realization of the connected components of $\beta_{\mathrm{s}}^{*} M$ ). Let $M \subset \mathbb{R}^{n}$ be a semialgebraic set. Then, there exists a semialgebraic compactification $X \subset \mathbb{R}^{m}$ of $M$ such that the number of connected components of $\partial M$ equals the number of connected components of $X \backslash M$.

The next example was inspiring for the proof of 5.8 .

Example 5.9. Consider the semialgebraic sets

$$
Q_{k}=\{-1 \leq x+k y \leq 1,-1 \leq x-k y \leq 1\}
$$

for $k=1,2$. Let $M=Q_{1} \backslash((-1,1) \times\{0\})$ and $M_{1}=\left(Q_{1} \backslash Q_{2}\right) \cup\{(-1,0),(1,0)\}$. Notice that $M$ and $M_{1}$ are semialgebraically homeomorphic; thus, $\beta_{\mathrm{s}}^{*} M \cong \beta_{\mathrm{s}}^{*} M_{1}$. By 5.2, the number of connected components of $\partial M \cong \partial M_{1}$, which is the number of connected components of $\mathrm{Cl}_{\mathbb{R}^{2}}\left(M_{1}\right) \backslash M_{1}$, is $\geq 2$. On the other hand, $M=C_{1} \cup C_{2}$, where each $C_{i}=M \cap\left\{(-1)^{i} y \geq 0\right\}$ is a closed subset of $M$. Notice that, by 3.14. $\partial M=\partial C_{1} \sqcup \partial C_{2}$ and, by 5.5, $\partial C_{i}$ is connected for $i=1,2$. Thus, the number of connected components of $\partial M$ is 2 and $X=\mathrm{Cl}_{\mathbb{R}^{2}}\left(M_{1}\right)$ is a semialgebraic compactification of $M$ whose remainder $X \backslash M_{1}$ has the same number of connected components as $\partial M$.

Proof of Theorem [5.8. We divide the proof into two steps. First, we claim:

Step 1. Let $C$ be a union of connected components of $\partial M$ and let $B=\partial M \backslash C$ be the union of the remaining connected components of $\partial M$. Then, there exists $f \in \mathcal{S}^{*}(M)$ such that $\left.\widehat{f}\right|_{C} \equiv 0$ and $\widehat{f}$ is strictly positive on $B$. 
Once this is done we will finish with:

Step 2. There exists a semialgebraic compactification $X \subset \mathbb{R}^{m}$ of $M$ such that the number of connected components of $\partial M$ equals the number of connected components of $X \backslash M$.

In fact, suppose for a while that Step 1 is proved and recall that $\partial M$ has, by [5.6. finitely many connected components, say $C_{1}, \ldots, C_{\ell}$. For each index $i=1, \ldots, \ell$, define $B_{i}=\bigcup_{j \neq i} C_{j}$. Since $C_{i}$ and $B_{i}$ satisfy the hypothesis of Step 1 there exists $f_{i} \in \mathcal{S}^{*}(M)$ such that $\left.\widehat{f}_{i}\right|_{C_{i}} \equiv 0$ and $\left.\widehat{f}_{i}\right|_{B_{i}}$ is strictly positive. By 4.1, there exist a semialgebraic compactification $X=\mathrm{Cl}_{\mathbb{R}^{n+\ell}}\left(\operatorname{graph}\left(f_{1}, \ldots, f_{\ell}\right)\right)$ of $M$ and for each index $i$ a semialgebraic extension $\widetilde{f}_{i}: X \rightarrow \mathbb{R}$ of $f_{i}$. By 4.6, there exists a surjective continuous map $\rho: \beta_{\mathrm{s}}^{*} M \rightarrow X$ which is the identity on $M$ and $\widehat{f}_{i}=\widetilde{f}_{i} \circ \rho$. Moreover, the restriction $\left.\rho\right|_{\partial M}: \partial M \rightarrow X \backslash M$ is proper and surjective, as we proved in 4.3, and so each image $\rho\left(C_{i}\right)$ is connected and closed in $X \backslash M$. Thus, by 5.2 all reduces to check that $\rho\left(C_{i}\right) \cap \rho\left(C_{j}\right)=\varnothing$ for $i \neq j$. Suppose there exists a common point $x \in \rho\left(C_{i}\right) \cap \rho\left(C_{j}\right)$. Then, $\tilde{f}_{i}(x)=0$ because $x \in \rho\left(C_{i}\right)$, but $\tilde{f}_{i}(x)>0$ since $x \in \rho\left(B_{i}\right)$, a contradiction.

(5.81) Therefore, all reduces to prove Step 1. First, by 2.1 we may assume as always that $M$ is bounded and, by 4.6, there exists a continuous surjective map $\rho: \beta_{\mathrm{s}}^{*} M \rightarrow X=\mathrm{Cl}_{\mathbb{R}^{n}}(M)$ which is the identity on $M$. By $4.3 \rho(\partial M)=X \backslash M$.

We apply 2.9 to the compact semialgebraic set $X=\mathrm{Cl}_{\mathbb{R}^{n}}(M)$ and the family of semialgebraic sets $\mathcal{F}=\left\{M, X \backslash M, M_{\mathrm{lc}}, Y=M \backslash M_{\mathrm{lc}}\right\}$, where $M_{\mathrm{lc}}$ is the largest dense and locally compact semialgebraic subset of $M$ (see 4.15). Thus, there exists a semialgebraic triangulation $(K, \Phi)$ of $X$ compatible with $\mathcal{F}$ such that, for each simplex $\sigma \in K$ which is not a face of other simplex of $K$, either $\sigma \subset \Phi^{-1}(M)$ or there exists a proper face $\tau$ of $\sigma$ satisfying $\tau^{0} \subset \sigma \backslash \Phi^{-1}(M) \subset \tau$.

To simplify notation we identify in what follows $X$ and the elements of $\mathcal{F}$ with their respective inverse images under $\Phi$. Note that $X$ is the union of the closed simplices $\sigma$ which are not a face of another simplex of $K$, and we denote them by $\sigma_{1}, \ldots, \sigma_{s}$.

For each $i=1, \ldots, s$, the intersection $S_{i}=\sigma_{i} \cap M$ is a closed subset of $M$ such that either $S_{i}=\sigma_{i}$ or $S_{i} \subsetneq \sigma_{i}$ and there exists a proper face $\tau_{i}$ of $\sigma_{i}$ with $\tau_{i}^{0} \subset \sigma_{i} \backslash M \subset \tau_{i}$. By 3.14 we may identify $\beta_{\mathrm{s}}^{*} S_{i} \equiv \mathrm{Cl}_{\beta_{\mathrm{s}}^{*} M}\left(S_{i}\right)$, and in this way, $S_{i}$ being closed in $M$, we get

$$
\partial S_{i}=\beta_{\mathrm{s}}^{*} S_{i} \backslash S_{i}=\beta_{\mathrm{s}}^{*} S_{i} \backslash M=\beta_{\mathrm{s}}^{*} S_{i} \cap\left(\beta_{\mathrm{s}}^{*} M \backslash M\right)=\beta_{\mathrm{s}}^{*} S_{i} \cap \partial M .
$$

Both intersections $C \cap \partial S_{i}$ and $B \cap \partial S_{i}$ are either empty or finite unions of connected components of $\partial S_{i}$, because $B$ and $C$ are open and closed subsets of $\partial M$. On the other hand, each $\partial S_{i}$ is, by 2.9 and [5.5. connected, because $\sigma_{i} \backslash S_{i}$ is connected too. Hence, for each index $i=1, \ldots, s$ either $C \cap \partial S_{i}=\varnothing$ or $C \cap \partial S_{i}=\partial S_{i}$, which is connected. Also the intersections $B \cap \partial S_{i}$ for $i=1, \ldots, s$ are either empty or coincide with $\partial S_{i}$, and, whenever $\partial S_{i}$ is nonempty, $B \cap \partial S_{i}=\partial S_{i}$ if and only if $C \cap \partial S_{i}=\varnothing$.

Consequently, we may assume that $C \cap \partial S_{i}=\partial S_{i} \neq \varnothing$ just for $i=1, \ldots, r \leq s$, $B \cap \partial S_{i}=\partial S_{i} \neq \varnothing$ just for $i=r+1, \ldots, t \leq s$ and $\partial S_{i}=\varnothing$ for $i=t+1, \ldots, s$. Moreover, $C=\bigcup_{i=1}^{r} \partial S_{i}$ and $B=\bigcup_{i=r+1}^{t} \partial S_{i}$.

(5.82) On the other hand, $\sigma_{i}^{0}$ is an open subset of $M$ for $i=1, \ldots, s$, because $\sigma_{i}$ is not a face of another simplex of $K$. In particular, since $M_{\mathrm{lc}}$ is dense in $M$ 
and $(K, \Phi)$ is compatible with $\mathcal{F}$, we deduce that $\sigma_{i}^{0} \subset M_{\mathrm{lc}}$ for $i=1, \ldots, s$. Thus, $Y_{i}=Y \cap S_{i}=S_{i} \backslash M_{\mathrm{lc}}$ is closed and nowhere dense in $S_{i}$ because it does not intersect $\sigma_{i}^{0}$. Hence, from 5.7 it follows that $\partial S_{i} \not \subset \mathrm{Cl}_{\beta_{\mathrm{s}}^{*} M}\left(Y_{i}\right)=\mathrm{Cl}_{\beta_{\mathrm{s}}^{*} M}\left(S_{i}\right) \cap \mathrm{Cl}_{\beta_{\mathrm{s}}^{*} M}(Y)$ for $i=1, \ldots, t$, where the last equality follows from 3.114

(5.8.3) After this preparation we introduce the numerical invariant

$$
\ell_{C}=\max \left\{\mathrm{ht}\left(\mathfrak{m}^{*}\right): \mathfrak{m}^{*} \in C\right\} \leq \operatorname{dim} M
$$

and we proceed by induction on $\ell_{C}$. Notice that, by [Fe1, 7.2], $\ell_{C} \geq 1$, and we are going to prove first that Step 1 holds true in case $\ell_{C}=1$.

[5.8 4) Fix $i=1, \ldots, r$ and let us see that $S_{i}$ has dimension 1.

By 5.812 there exists $\mathfrak{m}^{*} \in \partial S_{i} \backslash \mathrm{Cl}_{\beta_{\mathrm{s}}^{*} M}(Y) \subset C$. Then, $\rho\left(\mathfrak{m}^{*}\right)=p \in \sigma_{i} \backslash S_{i}$. Clearly, $\mathfrak{m}^{*} \notin \mathrm{Cl}_{\beta_{\mathrm{s}}^{*} M}(B)$ because $B$ and $C$ are closed disjoint subsets of $\partial M$. By 4.10, there exists $g \in \mathcal{S}^{*}(M)$ with $\widehat{g}\left(\mathfrak{m}^{*}\right)=1$ and $\left.\widehat{g}\right|_{\mathrm{Cl}_{\beta_{*}^{*} M}(B \cup Y)} \equiv 0$; let $\Gamma=\operatorname{graph}(g)$ and $X_{1}=\mathrm{Cl}_{\mathbb{R}^{n+1}}(\Gamma)$. By 4.1, there exists $\bar{g} \in \mathcal{S}^{*}\left(X_{1}\right)$ such that $g \circ\left(\left.\pi\right|_{\Gamma}\right)=\left.\bar{g}\right|_{\Gamma}$, where $\pi: \mathbb{R}^{n+1} \rightarrow \mathbb{R}^{n}$ is the projection onto the first $n$ coordinates. Since $X_{1}$ is a semialgebraic compactification of $M$ there exists, by 4.6, a continuous surjective map $\gamma: \beta_{\mathrm{s}}^{*} M \rightarrow X_{1}$ which is the identity on $M$. A straightforward computation shows that $\rho=\pi \circ \gamma$ (see 5.811). Then, the point $q=\gamma\left(\mathfrak{m}^{*}\right) \in X_{1}$ satisfies $\pi(q)=\pi\left(\gamma\left(\mathfrak{m}^{*}\right)\right)=\rho\left(\mathfrak{m}^{*}\right)=p$, and so $\operatorname{dim}_{q} X_{1} \geq \operatorname{dim}_{p} X$. Moreover, $\bar{g}(q)=$ $\bar{g}\left(\gamma\left(\mathfrak{m}^{*}\right)\right)=\widehat{g}\left(\mathfrak{m}^{*}\right)=1$ and

$$
q \in \gamma\left(\partial S_{i} \backslash \mathrm{Cl}_{\beta_{\mathrm{s}}^{*} M}(Y)\right) \subset \mathrm{Cl}_{X_{1}}\left(S_{i}\right) \backslash\left(S_{i} \cup \mathrm{Cl}_{X_{1}}(Y)\right) \subset X_{1} \backslash\left(M \cup \mathrm{Cl}_{X_{1}}(Y)\right) .
$$

By [Fe1, 7.1], there exists a maximal ideal $\mathfrak{n}^{*} \in \partial M$ such that $g \notin \mathfrak{n}^{*}$ and

$$
\operatorname{ht}\left(\mathfrak{n}^{*}\right)=\operatorname{dim}_{q} X_{1} \geq \operatorname{dim}_{p} X \geq \operatorname{dim}_{p} S_{i}=\operatorname{dim} S_{i} \geq 1 .
$$

On the other hand, $\mathfrak{n}^{*} \in C=\partial M \backslash B$, because $g \notin \mathfrak{n}^{*}$ and $\left.\widehat{g}\right|_{B} \equiv 0$. Whence $\operatorname{ht}\left(\mathfrak{n}^{*}\right) \leq \ell_{C}=1$, and this implies $\operatorname{dim} S_{i}=1$.

Once 5.814 is proved, we approach the proof of Step 1 in case $\ell_{C}=1$. Recall that we must construct a function $f \in \mathcal{S}^{*}(M)$ such that $\left.\widehat{f}\right|_{C} \equiv 0$ and $\left.\widehat{f}\right|_{B}$ is strictly positive. To that end, we write $M=M^{\geq 2} \cup L$, where $M^{\geq 2}$ is the set of points at which $M$ has local dimension $\geq 2$ (see 4.18$)$ and $L$ is the closure in $M$ of the set of points at which $M$ has local dimension $\leq 1$. In fact, $M^{\geq 2} \cap L$ is either empty or a finite set.

Since $M^{\geq 2}$ and $L$ are closed semialgebraic subsets of $M$, we may write, by using 3.14. $\beta_{\mathrm{s}}^{*} M=\mathrm{Cl}_{\beta_{\mathrm{s}}^{*} M}\left(M^{\geq 2}\right) \cup \mathrm{Cl}_{\beta_{\mathrm{s}}^{*} M}(L)=\beta_{\mathrm{s}}^{*} M^{\geq 2} \cup \beta_{\mathrm{s}}^{*} L$. Since $\operatorname{dim} S_{i}=1$ for $i=1, \ldots, r$ and each $\sigma_{i}$ is not a face of another simplex of $K$, we deduce that $S_{i} \subset L$ for $i=1, \ldots, r$. Thus, $C \subset \partial L$, and this last is a finite set (see 4.20) with, say, $c$ elements, that we denote $\partial L=\left\{\mathfrak{m}_{k}^{*}: 1 \leq k \leq c\right\}$. Moreover, by 3.114 (ii), we have

$$
\beta_{\mathrm{s}}^{*} M^{\geq 2} \cap \beta_{\mathrm{s}}^{*} L=\mathrm{Cl}_{\beta_{\mathrm{s}}^{*} M}\left(M^{\geq 2}\right) \cap \mathrm{Cl}_{\beta_{\mathrm{s}}^{*} M}(L)=\mathrm{Cl}_{\beta_{\mathrm{s}}^{*} M}\left(M^{\geq 2} \cap L\right)=M^{\geq 2} \cap L,
$$

because $M^{\geq 2} \cap L$ is a finite set. Hence, $\partial L \cap \beta_{\mathrm{s}}^{*} M^{\geq 2} \subset\left(M^{\geq 2} \cap L\right) \backslash L=\varnothing$ and so $C \subset \partial L \backslash \beta_{\mathrm{s}}^{*} M^{\geq 2}$. Since $L$ is a semialgebraic curve, $\beta_{\mathrm{s}}^{*} L$ is also a semialgebraic curve (see 4.20). In fact, one can check that for each point $\mathfrak{m}_{k}^{*} \in \partial L$ there exists a closed semialgebraic subset $A_{k} \subset L \backslash M^{\geq 2}=M \backslash M^{\geq 2}$ of $M$, which is homeomorphic to the interval $[0,1)$, and such that $\mathrm{Cl}_{\beta_{\mathrm{s}}^{*} M}\left(A_{k}\right)$ is a neighbourhood of $\mathfrak{m}_{k}^{*}$ in $\beta_{\mathrm{s}}^{*} M$. We may also assume that the neighbourhoods $A_{k}$ are pairwise disjoint. By 2.3 there 
exists $f \in \mathcal{S}^{*}(M)$ such that

$$
\left.f\right|_{M \geq 2} \equiv 1 \quad \text { and }\left.\quad f\right|_{A_{k}} \equiv \begin{cases}1 & \text { if } \mathfrak{m}_{k}^{*} \notin C \\ 0 & \text { if } \mathfrak{m}_{k}^{*} \in C\end{cases}
$$

Thus, $\left.\widehat{f}\right|_{B} \equiv 1>0$ and $\left.\widehat{f}\right|_{C} \equiv 0$. This finishes the proof of Step 1 in case $\ell_{C}=1$.

[5.8.5) Suppose that Step 1 is true for $\ell_{C} \leq d-1$ and let us see that it is also true for $\ell_{C}=d$.

Indeed, let $G=\bigcup_{i=1}^{r} \sigma_{i}^{0}$ which is an open subset of $M$. For each $i=1, \ldots, r$, consider the skeleton $\epsilon_{i}=\sigma_{i} \backslash \sigma_{i}^{0}$ of $\sigma_{i}$ and let $E_{i}=\epsilon_{i} \cap M=\epsilon_{i} \cap S_{i}$, which we call the skeleton of $S_{i}$. By the choice of the triangulation $(K, \Phi)$ in 5.811, $\operatorname{dim} E_{i}=\operatorname{dim} S_{i}-1$ for $i=1, \ldots, r$. Let us define the following closed semialgebraic subsets of $M$ :

$$
M_{1}=\bigcup_{i=1}^{r} S_{i}, \quad M_{2}=\bigcup_{i=r+1}^{t} S_{i} \text { and } M_{3}=\bigcup_{i=t+1}^{s} S_{i}=\bigcup_{i=t+1}^{s} \sigma_{i} .
$$

Notice that $M_{3}$ is compact and $\partial M=\partial M_{1} \cup \partial M_{2}$, with $C=\partial M_{1}$ and $B=\partial M_{2}$. Let $E=\bigcup_{i=1}^{r} E_{i} \subset M_{1}$ which is a closed subset of $M$ of $\operatorname{dimension} \operatorname{dim} E=$ $\operatorname{dim} M_{1}-1$. Thus,

$$
C_{1}=\partial E \subset \partial M_{1}=C \text { and } M_{0}=M \backslash G=E \cup M_{2} \cup M_{3} .
$$

Therefore, $\partial M_{0}=\partial E \cup \partial M_{2}=C_{1} \cup B$. Our next goal is to see that $\ell_{C_{1}}=$ $\ell_{C}-1$. To that end it suffices to prove that $\ell_{C}=\operatorname{dim} M_{1}$. Once this is done, and proceeding similarly with the suitable simplices which constitute $E$, one deduces $\ell_{C_{1}}=\operatorname{dim} E=\operatorname{dim} M_{1}-1=\ell_{C}-1$.

(5.8 6) We begin by proving that $\ell_{C} \leq \operatorname{dim} M_{1}$. Let $\mathfrak{m}^{*} \in C=\partial M_{1}$ be a maximal ideal of $\mathcal{S}^{*}(M)$. Then,

$$
\mathfrak{m}^{*} \notin \mathrm{Cl}_{\beta_{\mathrm{s}}^{*} M}\left(M_{2} \cup M_{3}\right)=M_{2} \cup M_{3} \cup B,
$$

because $M=M_{1} \cup M_{2} \cup M_{3}, \partial M_{3}=\varnothing$ and $B=\partial M_{2}$. By 4.10 there exists $g_{1} \in \mathcal{S}^{*}(M)$ such that $\left.\widehat{g}_{1}\right|_{\mathrm{Cl}_{\beta_{s}^{*} M}\left(M_{2} \cup M_{3}\right)} \equiv 0$ and $\widehat{g}_{1}\left(\mathfrak{m}^{*}\right)=1$. Let $\mathfrak{p}_{0} \subsetneq \cdots \subsetneq \mathfrak{p}_{\ell}=\mathfrak{m}^{*}$ be a chain of prime ideals of $\mathcal{S}^{*}(M)$ which does not admit refinements and such that $\ell=h t\left(\mathfrak{m}^{*}\right)$. For each $j=1, \ldots, \ell$, let $f_{j} \in \mathfrak{p}_{j} \backslash \mathfrak{p}_{j-1}$. By 4.1 there exist a semialgebraic compactification $X_{2}$ of $M$ and bounded semialgebraic functions $g_{2}, F_{j} \in \mathcal{S}^{*}\left(X_{2}\right)$ such that $\left.g_{2}\right|_{M}=g_{1}$ and each $\left.F_{j}\right|_{M}=f_{j}$. Therefore, the prime ideals $\mathfrak{q}_{i}=\mathfrak{p}_{i} \cap \mathcal{S}\left(X_{2}\right)$ satisfy $F_{j} \in \mathfrak{q}_{j} \backslash \mathfrak{q}_{j-1}$, and so there exists a chain of prime ideals $\mathfrak{q}_{0} \subsetneq \cdots \subsetneq \mathfrak{q}_{\ell}=\mathfrak{m}_{x}^{*}$ in $\mathcal{S}^{*}\left(X_{2}\right)$ for some point $x \in X_{2}$ such that $g_{2}(x) \neq 0$. This last is so because $\widehat{g_{1}}\left(\mathfrak{m}^{*}\right)=1$ implies $g_{1} \notin \mathfrak{m}^{*}$. Hence, $x \in X_{2} \backslash \mathrm{Cl}_{X_{2}}\left(M_{2} \cup M_{3}\right)$, because $\left.\widehat{g}_{1}\right|_{\mathrm{Cl}_{\beta_{\mathrm{s}}^{*} M}\left(M_{2} \cup M_{3}\right)} \equiv 0$. Moreover, from [FG2, 4.2], ht $\left(\mathfrak{m}_{x}^{*}\right)=\operatorname{dim}_{x} X_{2}$, and

$$
\begin{aligned}
& X_{2} \backslash \mathrm{Cl}_{X_{2}}\left(M_{2} \cup M_{3}\right) \\
& \quad=\mathrm{Cl}_{X_{2}}\left(M_{1} \cup M_{2} \cup M_{3}\right) \backslash \mathrm{Cl}_{X_{2}}\left(M_{2} \cup M_{3}\right)=\mathrm{Cl}_{X_{2}}\left(M_{1}\right) \backslash \mathrm{Cl}_{X_{2}}\left(M_{2} \cup M_{3}\right) .
\end{aligned}
$$

Consequently,

$$
\begin{aligned}
\operatorname{ht}\left(\mathfrak{m}^{*}\right) \leq \operatorname{ht}\left(\mathfrak{m}_{x}^{*}\right)=\operatorname{dim}_{x} X_{2}=\operatorname{dim}_{x}\left(X_{2} \backslash \mathrm{Cl}_{X_{2}}\left(M_{2} \cup M_{3}\right)\right) \\
\quad=\operatorname{dim}_{x}\left(\mathrm{Cl}_{X_{2}}\left(M_{1}\right) \backslash \mathrm{Cl}_{X_{2}}\left(M_{2} \cup M_{3}\right)\right) \leq \operatorname{dim} \mathrm{Cl}_{X_{2}}\left(M_{1}\right)=\operatorname{dim} M_{1},
\end{aligned}
$$

which implies $\ell_{C} \leq \operatorname{dim} M_{1}$.

5.87) We must now prove the converse inequality $\ell_{C} \geq \operatorname{dim} M_{1}$; hence, the equality $\ell_{C}=\operatorname{dim} M_{1}$. To that end it suffices to show the existence of a maximal 
ideal $\mathfrak{n}^{*} \in C$ such that $\operatorname{ht}\left(\mathfrak{n}^{*}\right)=\operatorname{dim} M_{1}$. After reordering the indices $i=1, \ldots, r$ we can suppose that $\operatorname{dim} M_{1}=\operatorname{dim} S_{1}$. Let us choose a point

$$
p_{1} \in \mathrm{Cl}_{\mathbb{R}^{n}}\left(S_{1}\right) \backslash S_{1} \subset \mathrm{Cl}_{\mathbb{R}^{n}}\left(\sigma_{1}^{0}\right) \backslash \sigma_{1}^{0} .
$$

By the Curve Selection Lemma BCR, 2.5.5] there exists a semialgebraic path $\alpha$ : $[0,1] \rightarrow \mathbb{R}^{n}$ such that $\alpha(0)=p_{1}$ and $\alpha((0,1]) \subset \sigma_{1}^{0}$. Note that as we observed in 5.8]2, $\sigma_{1}^{0} \cap Y=\varnothing$ and so $\alpha((0,1]) \subset M \backslash Y$. By 2.4 there exists $g_{3} \in \mathcal{S}^{*}(M)$ such that $g_{3} \circ \alpha \equiv 1$ and $\left.g_{3}\right|_{M \backslash \sigma_{1}^{0}} \equiv 0$. Thus,

$$
\mathfrak{m}_{\alpha}^{*} \in \beta_{\mathrm{s}}^{*} M \backslash\left(S_{1} \cup \mathrm{Cl}_{\beta_{\mathrm{s}}^{*} M}\left(M \backslash S_{1}\right) \cup \mathrm{Cl}_{\beta_{\mathrm{s}}^{*} M}(Y)\right) \subset \partial S_{1} \backslash \mathrm{Cl}_{\beta_{\mathrm{s}}^{*} M}(Y) .
$$

By 4.1 there exists a semialgebraic compactification $X_{3}=\mathrm{Cl}_{\mathbb{R}^{n+1}}\left(\operatorname{graph}\left(g_{3}\right)\right) \subset$ $\mathbb{R}^{n+1}$ of $M$ such that $g_{3}$ admits a semialgebraic extension to $X_{3}$. Using 2.3 once more there exists a semialgebraic extension $\overline{g_{3}}$ of $g_{3}$ to the whole $\mathbb{R}^{n+1}$. By 4.6, there exists a continuous surjective map $\gamma_{1}: \beta_{\mathrm{s}}^{*} M \rightarrow X_{3}$ which is the identity on $M$. Then, if $\pi: \mathbb{R}^{n+1} \rightarrow \mathbb{R}^{n}$ denotes the projection onto the first $n$ coordinates, $\rho=\pi \circ \gamma_{1}$ and the point $q_{1}=\gamma_{1}\left(\mathfrak{m}_{\alpha}^{*}\right) \in X_{3}$ satisfies $\pi\left(q_{1}\right)=p_{1}$. Hence, $\operatorname{dim}_{q_{1}} X_{3} \geq$ $\operatorname{dim}_{p_{1}} X$. Moreover, $\bar{g}_{3}\left(q_{1}\right)=\bar{g}_{3}\left(\gamma_{1}\left(\mathfrak{m}_{\alpha}^{*}\right)\right)=\widehat{g_{3}}\left(\mathfrak{m}_{\alpha}^{*}\right)=1$. Note that, $S_{1}$ being closed in $M$, we have $\mathrm{Cl}_{X_{3}}\left(S_{1}\right) \backslash S_{1} \subset X_{3} \backslash M$, and so

$$
q_{1} \in \gamma_{1}\left(\partial S_{1} \backslash \mathrm{Cl}_{\beta_{\mathrm{s}}^{*} M}(Y)\right) \subset \mathrm{Cl}_{X_{3}}\left(S_{1}\right) \backslash\left(S_{1} \cup \mathrm{Cl}_{X_{3}}(Y)\right) \subset X_{3} \backslash\left(M \cup \mathrm{Cl}_{X_{3}}(Y)\right) .
$$

By [Fe1, 7.1], there exists a maximal ideal $\mathfrak{n}^{*} \in \partial M$ such that $g_{3} \notin \mathfrak{n}^{*}$ and

$$
\operatorname{ht}\left(\mathfrak{n}^{*}\right)=\operatorname{dim}_{q_{1}} X_{3} \geq \operatorname{dim}_{p_{1}} X \geq \operatorname{dim}_{p} S_{1}=\operatorname{dim} S_{1}=\operatorname{dim} M_{1} .
$$

Since $g_{3} \notin \mathfrak{n}^{*}$, then $\mathfrak{n}^{*} \notin B \subset \mathrm{Cl}_{\beta_{\mathrm{s}}^{*} M}\left(M \backslash S_{1}\right) \subset \mathcal{Z}_{\beta_{\mathrm{s}}^{*} M}\left(\widehat{g_{3}}\right)$, and this implies $\mathfrak{n}^{*} \in C$. Thus,

$$
\operatorname{dim} M_{1} \leq \mathrm{ht}\left(\mathfrak{n}^{*}\right) \leq \ell_{C} \leq \operatorname{dim} M_{1}
$$

and we conclude $\ell_{C}=\operatorname{dim} M_{1}$, as claimed.

(5.8 8) Applying the inductive hypothesis to $C_{1}$ there exists $a \in \mathcal{S}(M \backslash G)$ such that $\left.\widehat{a}\right|_{C_{1}}=0$ and $\left.\widehat{a}\right|_{B}$ is strictly positive. Thus, it is enough to check that we can extend $a$ to a bounded semialgebraic function $b: M \rightarrow \mathbb{R}$ such that $\left.\widehat{b}\right|_{C} \equiv 0$.

The restriction $a_{i}=\left.a\right|_{E_{i}}$ admits, by 2.3, a semialgebraic extension $A_{i} \in \mathcal{S}^{*}\left(S_{i}\right)$. By 4.1, there is a semialgebraic compactification $T_{i}=\mathrm{Cl}_{\mathbb{R}^{n+1}}\left(\operatorname{graph}\left(A_{i}\right)\right)$ of $S_{i}$ to which we can extend $A_{i}$. Let us denote by $\widetilde{a_{i}}$ the semialgebraic extension of $a_{i}$ to $\mathrm{Cl}_{T_{i}}\left(E_{i}\right)$. Moreover, by the uniqueness of the continuous extensions, the function $\widehat{a_{i}}: \beta_{\mathrm{s}}^{*} E_{i} \rightarrow \mathbb{R}$ factorizes through $\mathrm{Cl}_{T_{i}}\left(E_{i}\right) \subset T_{i}$ via $\widetilde{a_{i}}$. Thus, $\widetilde{a_{i}}$ vanishes identically on $\mathrm{Cl}_{T_{i}}\left(E_{i}\right) \backslash E_{i}=\mathrm{Cl}_{T_{i}}\left(E_{i}\right) \backslash S_{i}$. Since $E_{i} \cup\left(T_{i} \backslash S_{i}\right)=T_{i} \backslash\left(S_{i} \backslash E_{i}\right)=T_{i} \backslash \sigma_{i}^{0}$ is a closed subset of $T_{i}$ and $\left.a_{i}\right|_{E_{i} \cap\left(T_{i} \backslash S_{i}\right)}=\left.a_{i}\right|_{E_{i} \backslash S_{i}} \equiv 0$, there exists, by 2.3, a semialgebraic function $G_{i} \in \mathcal{S}^{*}\left(T_{i}\right)$ such that $\left.G_{i}\right|_{E_{i}}=a_{i}$ and $\left.G_{i}\right|_{T_{i} \backslash S_{i}} \equiv 0$. Thus, extending $a$ by $\left.G_{i}\right|_{S_{i}}$ on each $S_{i}$ for $i=1, \ldots, r$, and since

$$
S_{i} \cap S_{j}=\sigma_{i} \cap \sigma_{j} \cap M=\epsilon_{i} \cap \epsilon_{j} \cap M=E_{i} \cap E_{j}
$$

for $1 \leq i<j \leq r$, we get the desired function $b \in \mathcal{S}^{*}(M)$ with the property that $\left.\widehat{b}\right|_{C}=0$. Since $b$ extends $a$, we also know that $\left.\widehat{b}\right|_{B}$ is strictly positive, and we are done.

(5.10) Connected components of $\partial M$ for locally compact $M$. Next, we explain how to compute the number of connected components of $\partial M$ for a locally compact semialgebraic set $M$ by means of its one point semialgebraic compactification. Since $M$ is locally compact, we may assume, by 4.17 , that $M \cup\{0\}$ is a compact 
set. Next, by [BCR, 9.3.6], there exist $\varepsilon>0$ and a semialgebraic homeomorphism $\varphi: \overline{\mathbb{B}}_{n}(0, \varepsilon) \rightarrow \overline{\mathbb{B}}_{n}(0, \varepsilon)$ such that:

(i) $\|\varphi(y)\|=\|y\|$ for every $y \in \overline{\mathbb{B}}_{n}(0, \varepsilon)$,

(ii) $\left.\varphi\right|_{\mathbb{S}^{n-1}(0, \varepsilon)}$ is the identity map,

(iii) $\varphi^{-1}\left(M \cap \overline{\mathbb{B}}_{n}(0, \varepsilon)\right)$ is the cone of vertex 0 and basis $M \cap \mathbb{S}^{n-1}(0, \varepsilon)$, with its vertex excluded.

Let us also denote $\varphi: \mathbb{R}^{n} \rightarrow \mathbb{R}^{n}$ as the extension of such homeomorphism to the whole $\mathbb{R}^{n}$ by using the identity map outside $\overline{\mathbb{B}}_{n}(0, \varepsilon)$, and we identify $M$ with $\varphi(M)$. After this preparatory work, and with the notation above, we enunciate:

Theorem 5.11. Let $M \subset \mathbb{R}^{n}$ be a locally compact semialgebraic set embedded in $\mathbb{R}^{n}$ as stated in 5.10 . Then, the number of connected components of $\partial M$ coincides with the number of connected components of $M \cap \mathbb{S}^{n-1}(0, \varepsilon)$, which also equals the number of connected components of the germ $M_{0}$ of $M$ at the origin of $\mathbb{R}^{n}$.

Before proving this, we need a preliminary result inspired in [GJ, 6.10] which is quite similar to 5.5 but simpler.

Lemma 5.12. Let $X \subset \mathbb{R}^{n}$ be a compact and connected semialgebraic subset of the unit sphere $\mathbb{S}^{n-1}(0,1)$. Let $Y_{0}=\left\{t x \in \mathbb{R}^{n}: x \in X\right.$ and $\left.t \in[0,1]\right\}$ be the cone of basis $X$ and vertex the origin of $\mathbb{R}^{n}$, and let $M=Y_{0} \backslash\{0\}$. Then, $\partial M$ is connected.

Proof. First note that $Y_{0}$ is the image of the continuous map

$$
[0,1] \times X \rightarrow \mathbb{R}^{n},(t, x) \mapsto t x .
$$

Hence, $Y_{0}$ is compact and therefore $M$ is locally compact. Thus, by $4.4 . M$ is open in $\beta_{\mathrm{s}}^{*} M$ and so $\partial M$ is a compact set. Suppose that $\partial M$ is not connected. Then, there exist two disjoint nonempty closed subsets $C_{1}, C_{2} \subset \partial M$ such that $\partial M=C_{1} \sqcup C_{2}$. Proceeding similarly to 5.511, there exists $h_{1} \in \mathcal{S}^{*}(M)$ whose unique extension $\widehat{h_{1}}: \beta_{\mathrm{s}}^{*} M \rightarrow \mathbb{R}$ to $\beta_{\mathrm{s}}^{*} M$ vanishes identically on $C_{1}$ and $\left.\widehat{h_{1}}\right|_{C_{2}} \equiv 1$. By [5.4, there exist two semialgebraic paths $\alpha_{1}, \alpha_{2}:[0,1] \rightarrow \mathbb{R}^{n}$ such that $\alpha_{i}((0,1]) \subset M, \alpha_{i}(0)=0$ and

$$
\lim _{s \rightarrow 0}\left(h_{1} \circ \alpha_{i}\right)(s)= \begin{cases}0 & \text { if } i=1, \\ 1 & \text { if } i=2 .\end{cases}
$$

Denote $X_{t}=\{t x: x \in X\}$ for each $t \in[0,1]$, which is a connected semialgebraic set since $X$ is as well. Thus, each $X_{t}$ is, by [BCR, 2.5.13], semialgebraically path connected. On the other hand, let $\delta>0$ such that im $\alpha_{i} \cap X_{t}$ is not empty for all $0<t<\delta$ and $i=1,2$. Fix $0<t<\delta$ and take $p_{i t} \in \operatorname{im} \alpha_{i} \cap X_{t}$ such that $\alpha_{i}(s) \in \bigcup_{\lambda \in(0, t)} X_{\lambda}$ for all $s \in\left(0, \alpha_{i}^{-1}\left(p_{i t}\right)\right)$.

Since $X_{t}$ is semialgebraically path connected, there exists a semialgebraic path $\nu_{t}:[0,1] \rightarrow X_{t}$ with $\nu_{t}(0)=p_{1 t}$ and $\nu_{t}(1)=p_{2 t}$. By suitably gluing the semialgebraic paths $\left.\alpha_{1}\right|_{\left(0, \alpha_{1}^{-1}\left(p_{1 t}\right)\right]}, \nu_{t}$ and $\left.\alpha_{2}\right|_{\left(0, \alpha_{2}^{-1}\left(p_{2 t}\right)\right]}$, we construct, for each $0<t<\delta$, a semialgebraic path $\gamma_{t}:(0,1) \rightarrow M$ such that

$$
\operatorname{im} \gamma_{t} \subset \bigcup_{\lambda \in(0, t)} X_{\lambda}, \quad \lim _{s \rightarrow 0}\left(h_{1} \circ \gamma_{t}\right)(s)=0, \quad \text { and } \quad \lim _{s \rightarrow 1}\left(h_{1} \circ \gamma_{t}\right)(s)=1 .
$$

Hence, for each $0<t<\delta$, there exists a point $q_{t} \in \bigcup_{\lambda \in(0, t)} X_{\lambda}$ such that $h_{1}\left(q_{t}\right)=$ $1 / 2$. Notice that for every $0<t<\delta$, we have $\left\|q_{t}\right\|=\lambda$ for some $0<\lambda<t$. By the compactness of $\beta_{\mathrm{s}}^{*} M$ there exists a cluster point $\mathfrak{m}^{*} \in \partial M$ of the set $\left\{q_{t}: 0<t<\delta\right\}$. 
Thus, $\widehat{h_{1}}\left(\mathfrak{m}^{*}\right)=1 / 2$, and this is a contradiction because $\left.\widehat{h_{1}}\right|_{\partial M}$ just attains the values 0 and 1 . Consequently, $\partial M$ is connected.

Proof of Theorem 5.11. We keep the notation introduced in statement 5.10. Let $X=\mathrm{Cl}_{\mathbb{R}^{n}}\left(M \backslash \overline{\mathbb{B}}_{n}(0, \varepsilon)\right)$, which is a compact set. Let $M_{1}, \ldots, M_{r}$ be the connected components of $M \cap \overline{\mathbb{B}}_{n}(0, \varepsilon)$. Note that each $M_{i} \cup\{0\}$ is a cone with vertex the origin and whose basis is a connected component of $M \cap \mathbb{S}^{n-1}(0, \varepsilon)$. Conversely, given a connected component $C_{i}$ of $M \cap \mathbb{S}^{n-1}(0, \varepsilon)$, let $K_{i}$ be the cone with vertex the origin and basis $C_{i}$. Then, $K_{i} \backslash\{0\}$ is a connected component of $M \cap \overline{\mathbb{B}}_{n}(0, \varepsilon)$ and we may assume that $K_{i}=M_{i}$ for $i=1, \ldots, r$.

We write $M=X \cup N$, where $N=M_{1} \cup \cdots \cup M_{r}$. Hence,

$$
\beta_{\mathrm{s}}^{*} M=\mathrm{Cl}_{\beta_{\mathrm{s}}^{*} M}(X) \cup \mathrm{Cl}_{\beta_{\mathrm{s}}^{*} M}(N) \text {. }
$$

Notice that, by 3.14, $\beta_{\mathrm{s}}^{*} X \cong \mathrm{Cl}_{\beta_{\mathrm{s}}^{*} M}(X)$ and $\beta_{\mathrm{s}}^{*} N \cong \mathrm{Cl}_{\beta_{\mathrm{s}}^{*} M}(N)$ because $X$ and $N$ are closed in $M$ and, since $X$ is compact, $\beta_{\mathrm{s}}^{*} X=X$. Therefore, after identifying $\beta_{\mathrm{s}}^{*} N$ with $\mathrm{Cl}_{\beta_{\mathrm{s}}^{*} M}(N)$, we have $\beta_{\mathrm{s}}^{*} M=X \cup \beta_{\mathrm{s}}^{*} N$, and since $\mathrm{Cl}_{\beta_{\mathrm{s}}^{*} M}(N) \cap M=N$,

$$
\partial M=\beta_{\mathrm{s}}^{*} M \backslash M=\left(X \cup \beta_{\mathrm{s}}^{*} N\right) \backslash M=\beta_{\mathrm{s}}^{*} N \backslash N=\partial N .
$$

Now, by 3.15 $\beta_{\mathrm{s}}^{*} N \cong \beta_{\mathrm{s}}^{*} M_{1} \sqcup \cdots \sqcup \beta_{\mathrm{s}}^{*} M_{r}$, and therefore

$$
\partial M \cong\left(\beta_{\mathrm{s}}^{*} M_{1} \backslash M_{1}\right) \sqcup \cdots \sqcup\left(\beta_{\mathrm{s}}^{*} M_{r} \backslash M_{r}\right)=\partial M_{1} \sqcup \cdots \sqcup \partial M_{r} .
$$

Each $M_{i}$ is locally compact since it is a closed subset of the locally compact space $N$. Thus, $\partial M_{i}$ is compact and therefore it is closed in $\partial M$. Consequently, the disjoint sets $\partial M_{i}$ are open and closed in $\partial M$. Hence, the connected components of $\partial M$ are the collection of all connected components of the sets $\partial M_{i}$. Thus, all reduces to check that each $\partial M_{i}$ is connected. This follows at once from 5.12 because $M_{i} \cup\{0\}$ is the cone with vertex the origin and basis a connected component of $M \cap \mathbb{S}^{n-1}(0, \varepsilon)$.

Once we know that the number of connected components of the remainder $\partial M$ is finite (see 5.6), we are ready to prove that it is also locally connected. Moreover, we will also determine under what conditions on $M$ the remainder $\partial M$ is locally compact.

(5.13) Local connectedness and local compactness of $\partial M$. Let $M \subset \mathbb{R}^{n}$ be a semialgebraic set. We begin by proving the local connectedness of $\partial M$.

Proof. Let $\mathfrak{m}^{*} \in \partial M$ and let $V$ be an open neighbourhood of $\mathfrak{m}^{*}$ in $\partial M$. Since $\beta_{\mathrm{s}}^{*} M$ is locally compact, there exists $f \in \mathcal{S}^{*}(M)$ such that

$$
\mathfrak{m}^{*} \in \mathcal{D}_{\beta_{\mathrm{s}}^{*} M}(f) \cap \partial M \subset \mathrm{Cl}_{\beta_{\mathrm{s}}^{*} M}\left(\mathcal{D}_{\beta_{\mathrm{s}}^{*} M}(f)\right) \cap \partial M \subset V .
$$

Moreover, as one can check almost straightforwardly,

$$
\mathrm{Cl}_{\beta_{\mathrm{s}}^{*} M}\left(\mathcal{D}_{\beta_{\mathrm{s}}^{*} M}(f)\right)=\mathrm{Cl}_{\beta_{\mathrm{s}}^{*} M}\left(D_{M}(f)\right) .
$$

Next, consider the semialgebraic set $T=\mathrm{Cl}_{M}\left(D_{M}(f)\right)=\mathrm{Cl}_{\beta_{\mathrm{s}}^{*} M}\left(D_{M}(f)\right) \cap M$. By 3.14, $\beta_{\mathrm{s}}^{*} T=\mathrm{Cl}_{\beta_{\mathrm{s}}^{*} M}(T) \subset \beta_{\mathrm{s}}^{*} M$. Moreover, since $T$ is closed in $M, \mathrm{Cl}_{\beta_{\mathrm{s}}^{*} M}(T) \cap M=T$ and so

$$
\partial T=\beta_{\mathrm{s}}^{*} T \backslash T=\mathrm{Cl}_{\beta_{\mathrm{s}}^{*} M}(T) \backslash M=\mathrm{Cl}_{\beta_{\mathrm{s}}^{*} M}(T) \cap \partial M=\mathrm{Cl}_{\beta_{\mathrm{s}}^{*} M}\left(D_{M}(f)\right) \cap \partial M
$$

has, by 5.6. finitely many connected components, $C_{1}, \ldots, C_{r}$, which are therefore open and closed subsets in $\partial T$. Let $C_{1} \subset \partial T$ be the connected component of $\partial T$ 
containing $\mathfrak{m}^{*}$. Since $\partial T=\mathrm{Cl}_{\beta_{\mathrm{s}}^{*} M}(T) \cap \partial M$ is closed in $\partial M$, the union $C=\bigcup_{i=2}^{r} C_{i}$ is a closed subset of $\partial M$ such that $\mathfrak{m}^{*} \notin C$. Notice that

$$
W=\left(\mathcal{D}_{\beta_{\mathrm{s}}^{*} M}(f) \cap \partial M\right) \backslash C \subset\left(\mathrm{Cl}_{\beta_{\mathrm{s}}^{*} M}\left(D_{M}(f)\right) \cap \partial M\right) \backslash C=\partial T \backslash C=C_{1} \subset V,
$$

and $W$ is an open neighbourhood of $\mathfrak{m}^{*}$ in $\partial M$. Hence, $C_{1}$ is a connected neighbourhood of $\mathfrak{m}^{*}$ contained in $V$, which proves that $\partial M$ is locally connected.

We now determine under what conditions $\partial M$ is locally compact. Indeed, with the notation already introduced in 4.15, we have

Proposition 5.14. Let $M \subset \mathbb{R}^{n}$ be a semialgebraic set. Then, $\partial M$ is locally compact if and only if $\rho_{1}(M)$ is compact.

Proof. Recall first that, by 4.15 the set $\rho_{1}(M)$ does not depend on the immersion of $M$ in $\mathbb{R}^{n}$, because $\rho_{1}(M)=M \backslash M_{\mathrm{lc}}$ and $M_{\mathrm{lc}}$ equals the set of points of $M$ which have a compact neighbourhood in $M$. Hence, we may assume, by 2.1 that $M$ is bounded. Let $X=\mathrm{Cl}_{\mathbb{R}^{n}}(M)$, which is a semialgebraic compactification of $M$. Thus, by 4.6, there exists a surjective continuous map $\rho: \beta_{\mathrm{s}}^{*} M \rightarrow X$ which is the identity on $M$. By [3.14] its restriction $\left.\rho\right|_{\partial M}: \partial M \rightarrow X \backslash M$ is proper and surjective.

Now, if $\partial M$ is locally compact, then $\rho_{0}(M)=X \backslash M=\rho(\partial M)$ is, by [Bo, Cor. $\S 10.4$, page 106], locally compact too. Thus, by 4.15 $\rho_{1}(X \backslash M)=\rho_{1}\left(\rho_{0}(M)\right)=\varnothing$. Therefore,

$$
\mathrm{Cl}_{\mathbb{R}^{n}}\left(\rho_{1}(M)\right) \backslash \rho_{1}(M)=\rho_{0}\left(\rho_{1}(M)\right)=\rho_{0}\left(\rho_{0}\left(\rho_{0}(M)\right)\right)=\rho_{1}\left(\rho_{0}(M)\right)=\varnothing,
$$

and so $\rho_{1}(M) \subset X$ is closed in $\mathbb{R}^{n}$. Since $X$ is compact, $\rho_{1}(M)$ is compact too.

Conversely, suppose that $Y=\rho_{1}(M)$ is compact and write $M_{\mathrm{lc}}=M \backslash Y$, which is, by 4.15 locally compact. By $4.14 \partial M_{\mathrm{lc}}$ is a closed subset of the compact space $\beta_{\mathrm{s}}^{*} M_{\mathrm{lc}}$. Thus, $\partial M_{\mathrm{lc}}$ is compact, and so it is locally compact. Moreover, by 3.114 . $\mathrm{Cl}_{\beta_{\mathrm{s}}^{*} M}(Y)=\beta_{\mathrm{s}}^{*} Y=Y$ because $Y$ is compact. Denote $j: M_{\mathrm{lc}} \hookrightarrow M$ as the inclusion map. By [FG3, 6.7] the map $\left.\beta_{\mathrm{s}}^{*} j\right|_{\beta_{\mathrm{s}}^{*} M_{\mathrm{lc}} \backslash\left(\beta_{\mathrm{s}}^{*} j\right)^{-1}(Y)}: \beta_{\mathrm{s}}^{*} M_{\mathrm{lc}} \backslash\left(\beta_{\mathrm{s}}^{*} j\right)^{-1}(Y) \rightarrow \beta_{\mathrm{s}}^{*} M \backslash Y$ is a homeomorphism. Thus, $\partial M=\left(\beta_{\mathrm{s}}^{*} M \backslash Y\right) \backslash M_{\mathrm{lc}}$ is homeomorphic to

$$
\left(\beta_{\mathrm{s}}^{*} M_{\mathrm{lc}} \backslash M_{\mathrm{lc}}\right) \backslash\left(\beta_{\mathrm{s}}^{*} j\right)^{-1}(Y)=\partial M_{\mathrm{lc}} \backslash\left(\beta_{\mathrm{s}}^{*} j\right)^{-1}(Y),
$$

which is locally compact because it is an open subset of the locally compact space $\partial M_{\mathrm{lc}}$. Consequently, $\partial M$ is locally compact.

Remark 5.15. Let $M \subset \mathbb{R}^{n}$ be a semialgebraic set. Then, $\partial M$ is compact if and only if $M$ is open in $\beta_{\mathrm{s}}^{*} M$ or, equivalently, if $M$ is locally compact (see 4.14).

(5.16) Nonsemialgebraicity of $\beta_{\mathrm{s}}^{*} M$. Our next purpose is to characterize those semialgebraic sets whose semialgebraic Stone-Čech compactification is homeomorphic to a semialgebraic set, in this way completing 4.19. We show that this happens very rarely.

Proposition 5.17. Let $M \subset \mathbb{R}^{n}$ be a semialgebraic set and let $\beta_{\mathrm{s}}^{*} M$ be its semialgebraic Stone-Čech compactification. The following assertions are equivalent:

(i) The semialgebraic set $M^{\geq 2}$ is compact.

(ii) The remainder $\partial M=\beta_{\mathrm{s}}^{*} M \backslash M$ is a finite set.

(iii) $\beta_{\mathrm{s}}^{*} M$ is homeomorphic to a semialgebraic set.

(iv) $\beta_{\mathrm{s}}^{*} M$ is metrizable. 
Proof. The implication (i) $\Longrightarrow$ (ii) follows from 4.19 and (iii) $\Longrightarrow$ (iv) is immediate.

(ii) $\Longrightarrow$ (iii) By 5.8, there exists a semialgebraic compactification $X$ of $M$ such that the number of connected components of $\partial M$ equals the number of connected components of $X \backslash M$. By 4.6, there exists a continuous map $\rho: \beta_{\mathrm{s}}^{*} M \rightarrow X$ such that $\left.\rho\right|_{M}=\operatorname{id}_{M}$ and, by $4.3(\mathrm{i}), X \backslash M=\rho(\partial M)$ is a finite set, because so is $\partial M$. Since the (finite) number of connected components (and so the number of elements) of both sets $\partial M$ and $X \backslash M$ coincide and $\rho(\partial M)=X \backslash M$, the restriction $\left.\rho\right|_{\partial M}: \partial M \rightarrow X \backslash M$ is a bijection. Hence, $\rho: \beta_{\mathrm{s}}^{*} M \rightarrow X$ is a continuous bijection between a compact set and a Hausdorff space, that is, $\rho$ is a homeomorphism. Therefore, $\beta_{\mathrm{s}}^{*} M$ is homeomorphic to the semialgebraic set $X$.

(iv) $\Longrightarrow$ (i) Let us prove that if $M^{\geq 2}$ is not compact, then $\beta_{\mathrm{s}}^{*} M$ is not metrizable. As is well known, to prove that a separable space is not metrizable just requires checking that its topology does not admit a countable basis. So it is convenient in our case to first show the separability of $\beta_{\mathrm{s}}^{*} M$. Since $M$ is dense in $\beta_{\mathrm{s}} M$, it suffices to prove that $M$ is separable. By [BCR, 2.9.10], $M$ is the disjoint union of a finite number of Nash submanifolds $N$, each of them Nash diffeomorphic to an open hypercube $(0,1)^{\operatorname{dim} N}$. Hence, since each hypercube is separable, the same holds for each $N$ and so for $M$.

Suppose now, by way of contradiction, that $\beta_{\mathrm{s}}^{*} M$ is metrizable, and so it admits a countable basis. By 2.1, we may assume that $M$ is bounded, and there exists, by 4.16, a point

$$
p \in \mathrm{Cl}_{\mathbb{R}^{n}}\left(M^{\geq 2}\right) \backslash\left(\mathrm{Cl}_{\mathbb{R}^{n}}\left(\rho_{1}\left(M^{\geq 2}\right)\right) \cup M^{\geq 2}\right) .
$$

Observe that $p \notin M$, because $M^{\geq 2}$ is closed in $M$, and denote $X=\mathrm{Cl}_{\mathbb{R}^{n}}(M)$. By 2.6. there exists semialgebraic triangulation $(K, \Phi)$ of $X$ compatible with the family $\mathcal{F}=\{M, X \backslash M,\{p\}\}$. We identify $X$ with $|K|, M$ with $\Phi^{-1}(M)$ and $p$ with $\Phi^{-1}(p)$. Let $\sigma$ be a simplex of $K$ of dimension $d \geq 2$ such that $p$ is one of its vertices and $\sigma^{0}$ is contained in $M$. We may assume that $p$ is the origin and that $\sigma \backslash\{p\} \subset\left\{x_{d}>0, x_{d+1}=0, \ldots, x_{n}=0\right\}$. Consider the noncountable set

$$
S=\left\{a \in \mathbb{R}^{d-1}: \exists \varepsilon>0 \mid(a t, t, 0, \ldots, 0) \in \sigma \quad \forall t \in[0, \varepsilon]\right\},
$$

and define, for each $a \in S$, the maximal ideal of $\mathcal{S}^{*}(M)$ given by

$$
\mathfrak{m}_{a}^{*}=\left\{f \in \mathcal{S}^{*}(M): \lim _{t \rightarrow 0} f(a t, t, 0, \ldots, 0)=0\right\}
$$

(see 5.3 . Let us see that for all $a \in S$ there exists $f_{a} \in \mathcal{S}^{*}(M)$ such that $\widehat{f_{a}}\left(\mathfrak{m}_{a}^{*}\right)<0$ and $\widehat{f}_{a}\left(\mathfrak{m}_{b}^{*}\right)>0$ for all $b \in S \backslash\{a\}$. Let

$$
f_{a}(x)=\max \left\{-1, \frac{\left(x_{1}-a_{1} x_{d}\right)^{2}+\cdots+\left(x_{d-1}-a_{d-1} x_{d}\right)^{2}-x_{d}^{4}}{\left(x_{1}-a_{1} x_{d}\right)^{2}+\cdots+\left(x_{d-1}-a_{d-1} x_{d}\right)^{2}+x_{d}^{4}+\cdots+x_{n}^{4}}\right\} .
$$

A straightforward computation shows that $\left|f_{a}\right| \leq 1$ and therefore $f_{a} \in \mathcal{S}^{*}(M)$. On the other hand, $\widehat{f}_{a}\left(\mathfrak{m}_{a}^{*}\right)=-1<0$ and $\widehat{f}_{a}\left(\mathfrak{m}_{b}^{*}\right)=1>0$ for all $b \neq a$.

Consider the family of open sets $U_{a}=\mathcal{U}_{\beta_{\mathrm{s}}^{*} M}\left(-f_{a}\right)$ in $\beta_{\mathrm{s}}^{*} M$. Note that $\mathfrak{m}_{a}^{*} \in U_{a}$ but $\mathfrak{m}_{b}^{*} \notin U_{a}$ for $b \neq a$. Let $\mathcal{B}=\left\{B_{m}\right\}_{m \geq 1}$ be a countable basis of the topology of $\beta_{\mathrm{s}}^{*} M$. For each $a \in S$ there exists $m_{a} \geq 1$ such that $\mathfrak{m}_{a}^{*} \in B_{m_{a}} \subset U_{a}$, and since $\mathcal{B}$ is countable but $S$ is not countable, there exist distinct points $a_{1}, a_{2} \in S$ such that $m_{a_{1}}=m_{a_{2}}$. Thus, $\mathfrak{m}_{a_{1}}^{*} \in B_{m_{a_{1}}}=B_{m_{a_{2}}} \subset U_{a_{2}}$, a contradiction, and so $\beta_{\mathrm{s}}^{*} M$ is not metrizable. 
We have characterized in 5.17 the semialgebraic sets $M$ whose semialgebraic Stone-Čech compactification $\beta_{\mathrm{s}}^{*} M$ is metrizable. The proof heavily relies on the separability of $\beta_{\mathrm{s}}^{*} M$, which is an immediate consequence of the separability of $M$. A natural question is to decide the metrizability of the remainder $\partial M$. In this direction a first step should be to answer the following question whose expected answer is stated just below in 5.19

Question 5.18. Under which assumptions is the remainder $\partial M$ a separable space?

$$
\begin{aligned}
& \text { Let } M \subset \mathbb{R}^{n} \text { be a semialgebraic set. Then, } \\
& \beta_{\mathrm{s}}^{*} M \text { is metrizable } \Longleftrightarrow \partial M \text { is finite } \Longleftrightarrow \partial M \text { is separable. }
\end{aligned}
$$

The first equivalence in 5.19 is proved in 5.17 and the difficult point is to prove that the separability of $\partial M$ implies one (and so both) of the other conditions.

The next step should be to study the metrizability of the remainder $\partial M$. In the absence of the separability assumption, to analyze the metrizability of $\partial M$ one is forced to use the classical Nagata-Smirnov metrizability theorem (see [Mu, §40]). Such a result claims that the regularity of a topological space and the existence of a countable and locally finite basis of its topology are equivalent to its metrizability. Hence, the strategy used in 5.17 to approach the metrizability of $\beta_{\mathrm{s}}^{*} M$ no longer works and new ideas are needed. However, we expect that the following statement holds.

$$
\begin{aligned}
& \text { Let } M \subset \mathbb{R}^{n} \text { be a semialgebraic set. Then, } \\
& \beta_{\mathrm{s}}^{*} M \text { is metrizable } \Longleftrightarrow \partial M \text { is metrizable } \Longleftrightarrow \partial M \text { is finite. }
\end{aligned}
$$

To finish we study under what conditions $\beta_{\mathrm{s}}^{*}$ commutes with finite products, in the same vein as [GJ], §6].

Proposition 5.21. Let $N \subset \mathbb{R}^{n}$ and $M \subset \mathbb{R}^{m}$ be semialgebraic sets. Then, the product $\beta_{\mathrm{s}}^{*} M \times \beta_{\mathrm{s}}^{*} N$ is homeomorphic to $\beta_{\mathrm{s}}^{*}(M \times N)$ if and only if either $M$ or $N$ are finite sets or both $M$ and $N$ are compact.

Proof. $\Longleftarrow)$ Since the roles of $M$ and $N$ are interchangeable, we begin by proving that if $N$ is finite, say with $k$ elements, then $\beta_{\mathrm{s}}^{*} M \times \beta_{\mathrm{s}}^{*} N \cong \beta_{\mathrm{s}}^{*}(M \times N)$. Note that $M \times N$ is semialgebraically homeomorphic to $M \times\{1, \ldots, k\}$. Thus, the product $M \times N \cong \bigsqcup_{j=1}^{k} M \times\{j\}$ and we deduce, from 3.115 and 4.4, that

$$
\beta_{\mathrm{s}}^{*}(M \times N)=\beta_{\mathrm{s}}^{*}\left(\bigsqcup_{j=1}^{k} M \times\{j\}\right) \cong \bigsqcup_{j=1}^{k} \beta_{\mathrm{s}}^{*} M \times\{j\}=\beta_{\mathrm{s}}^{*} M \times N=\beta_{\mathrm{s}}^{*} M \times \beta_{\mathrm{s}}^{*} N .
$$

On the other hand, if $M$ and $N$ are compact, then $M \times N$ is compact too and, by 4.4 $\beta_{\mathrm{s}}^{*}(M \times N) \cong M \times N \cong \beta_{\mathrm{s}}^{*} M \times \beta_{\mathrm{s}}^{*} N$.

$\Longrightarrow)$ For the converse, we may assume that $M$ and $N$ are infinite sets and $M$ is bounded (by 2.1) but not compact. Let us see that $\beta_{\mathrm{s}}^{*} M \times \beta_{\mathrm{s}}^{*} N \neq \beta_{\mathrm{s}}^{*}(M \times N)$. Indeed, since $M$ is not compact, it contains, by the Curve Selection Lemma BCR, 2.5.5], a semialgebraic set $C_{1} \subset M$ semialgebraically homeomorphic to $(0,1]$ which is closed in $M$. Also, since $\operatorname{dim} N \geq 1$, there exists, again by the Curve Selection Lemma [BCR, 2.5.5], a closed semialgebraic set $C_{2} \subset N$ which is semialgebraically 
homeomorphic to $[0,1]$. The set $C_{1} \times C_{2}$ is a closed subset of $M \times N$. Suppose that $\beta_{\mathrm{s}}^{*} M \times \beta_{\mathrm{s}}^{*} N \cong \beta_{\mathrm{s}}^{*}(M \times N)$. Then, by $3.114(\mathrm{ii})$,

$$
\begin{aligned}
\beta_{\mathrm{s}}^{*}\left(C_{1} \times C_{2}\right) \cong \mathrm{Cl}_{\beta_{\mathrm{s}}^{*}(M \times N)}\left(C_{1} \times C_{2}\right) & \cong \mathrm{Cl}_{\beta_{\mathrm{s}}^{*} M \times \beta_{\mathrm{s}}^{*} N}\left(C_{1} \times C_{2}\right) \\
& =\mathrm{Cl}_{\beta_{\mathrm{s}}^{*} M}\left(C_{1}\right) \times \mathrm{Cl}_{\beta_{\mathrm{s}}^{*} N}\left(C_{2}\right) \cong \beta_{\mathrm{s}}^{*} C_{1} \times \beta_{\mathrm{s}}^{*} C_{2} .
\end{aligned}
$$

By $4.9, \beta_{\mathrm{s}}^{*} C_{i} \cong[0,1]$. Therefore,

$$
\beta_{\mathrm{s}}^{*}\left(C_{1} \times C_{2}\right) \cong \beta_{\mathrm{s}}^{*} C_{1} \times \beta_{\mathrm{s}}^{*} C_{2} \cong[0,1]^{2} .
$$

In particular, $\beta_{\mathrm{s}}^{*}\left(C_{1} \times C_{2}\right)$ is homeomorphic to a semialgebraic set. Hence, by 5.17, the set $C_{1} \times C_{2} \cong(0,1] \times[0,1]$ of points of $C_{1} \times C_{2}$ of local dimension $\geq 2$ is compact, a contradiction. Therefore, $\beta_{\mathrm{s}}^{*} M \times \beta_{\mathrm{s}}^{*} N \not \beta_{\mathrm{s}}^{*}(M \times N)$.

\section{REFERENCES}

[BCR] J. Bochnak, M. Coste, M.-F. Roy: Real algebraic geometry. Ergeb. Math. 36, SpringerVerlag, Berlin, 1998. MR1659509 (2000a:14067)

[Bo] N. Bourbaki: General topology. Chapters 1-4. Elements of Mathematics. Springer-Verlag, Berlin, 1989. MR979294(90a:54001a)

[DK] H. Delfs, M. Knebusch: Separation, retractions and homotopy extension in semialgebraic spaces. Pacific J. Math. 114 (1984), no. 1, 47-71. MR755482 (85i:14016)

[Fe1] J.F. Fernando: On chains of prime ideals in rings of semialgebraic functions. Preprint $R A A G$ (2010). http://www.mat.ucm.es/ josefer/pdfs/preprint/chains.pdf

$[\mathrm{Fe} 2]$ J.F. Fernando: On distinguished points of the remainder of the semialgebraic Stone-Čech compactification of a semialgebraic set. Preprint RAAG (2010). http://www.mat.ucm.es/ josefer/pdfs/preprint/remainder.pdf

[FG1] J.F. Fernando, J.M. Gamboa: On Łojasiewicz's inequality and the Nullstellensatz for rings of semialgebraic functions. Preprint RAAG (2010). http://www.mat. ucm.es/ josefer/pdfs/preprint/null-loj.pdf

[FG2] J.F. Fernando, J.M. Gamboa: On the Krull dimension of rings of semialgebraic functions. Preprint RAAG (2010). http://www.mat.ucm.es/ josefer/pdfs/preprint/dim.pdf

[FG3] J.F. Fernando, J.M. Gamboa: On the spectra of rings of semialgebraic functions. Collect. Math., to appear (2012).

[FG4] J.F. Fernando, J.M. Gamboa: On Banach-Stone type theorems in the semialgebraic setting. Preprint RAAG (2010). http://www.mat.ucm.es/ josefer/pdfs/ preprint/homeo.pdf

[GJ] L. Gillman, M. Jerison: Rings of continuous functions. The Univ. Series in Higher Nathematics 1, D. Van Nostrand Company, Inc., 1960. MR0116199(22:6994)

[MO] G. De Marco, A. Orsatti: Commutative rings in which every prime ideal is contained in a unique maximal ideal. Proc. Amer. Math. Soc. 30 (1971), no 3, 459-466. MR0282962 $(44: 196)$

[Mu] J.R. Munkres: Topology: A first course. Prentice-Hall, Inc., Englewood Cliffs, New Jersey, 1975. MR0464128(57:4063)

Departamento de Álgebra, Facultad de Ciencias Matemáticas, Universidad ComPLUTEnse de MAdRid, 28040 Madrid, Spain

E-mail address: josefer@mat.ucm.es

Departamento de Álgebra, Facultad de Ciencias Matemáticas, Universidad ComPlutense de Madrid, 28040 MAdrid, SPAin

E-mail address: jmgamboa@mat.ucm.es 